\title{
LA DEMANDA TURÍSTICA EN UNA CADENA HOTELERA. SERIES TEMPORALES PARA UN MODELO DE PREDICCIÓN
}

THE TOURIST DEMAND OF THE HOTEL CHAIN. TIME SERIES FOR A FORECAST MODEL

\author{
Reinier Fernández López \\ Universidad de Pinar del Río Hermanos \\ Saíz Monte de Oca \\ Pinar del Río, Pinar del Río, Cuba \\ rflopez@upr.edu.cu \\ Arely Quintero Silverio \\ Universidad de Pinar del Río Hermanos \\ Saíz Monte de Oca \\ Pinar del Río, Pinar del Río, Cuba \\ arelys@upr.edu.cu
}

\author{
José Alberto Vilalta Alonso \\ Universidad Tecnológica de la Habana \\ José Antonio Echeverría \\ La Habana, La Habana, Cuba \\ jvilalta@ind.cujae.edu.cu \\ Ledy Díaz González \\ Universidad de Pinar del Río Hermanos \\ Saíz Monte de Oca \\ Pinar del Río, Pinar del Río, Cuba \\ ledy@upr.edu.cu
}

Fecha de recepción: 12/08/2020 - Fecha de revisión: 07/10/2020 - Fecha de aprobación: 15/12/2020 DOI: https://doi.org/10.36995/j.visiondefuturo.2021.25.01.004.es

\section{RESUMEN}

En un mundo cada vez más incierto donde la dinámica mundial acelera la forma de gestionar los procesos en cualquier sector cobra mucha importancia el pronóstico de la demanda turística. En tal sentido, la presente investigación tiene como objetivo pronosticar la demanda turística de la Cadena Hotelera Cubanacán de Pinar del Río, Cuba, hasta diciembre del año 2019, mediante el empleo de técnicas de series cronológicas, que facilite la planificación y la toma de decisiones en este sector y de este modo trabajar por el logro de una integración en las cadenas productivas, considerando que el turismo es una de las actividades socioeconómicas que activa muchos otros sectores de la producción y los servicios, así como predecir el comportamiento del turismo. Para ello, se empleó el método cuantitativo de investigación como método rector, sustentado en la metodología Box - Jenkins y el método de alisado exponencial de Holt - Winters. También se logra caracterizar la gestión turística tomando en consideración dos indicadores: el costo por peso y los ingresos medios por turista, haciendo referencia a la eficiencia y eficacia respectivamente. Se realizó, además, un análisis multivariado de series temporales que posibilitó caracterizar la actividad turística en cuatro etapas fundamentales en la cadena hotelera tomada como objeto de estudio.

PALABRAS CLAVE: Demanda turística; Eficacia; Eficiencia; Series temporales.

\section{ABSTRACT}

In an increasingly uncertain world where world dynamics accelerates the way of "Visión de Futuro" Año 18, Volumen N²5 N 1, Enero - Junio 2021 - Pág 107-132 URL de la Revista: http://visiondefuturo.fce.unam.edu.ar/index.php/visiondefuturo/index URL del Documento: https://visiondefuturo.fce.unam.edu.ar/index.php/visiondefuturo/issue/view/19 ISSN 1668 - 8708 - Versión en Línea 
managing processes in any sector, the forecast of tourist demand becomes very important. In this sense, the present research aims to forecast the tourist demand of the Cuban Hotel Chain of Pinar del Río, Cuba, until December 2019, through the use of time series techniques, which facilitate planning and decision-making. in this sector and in this way work towards the achievement of an integration in the productive chains, considering that tourism is one of the socioeconomic activities that activates many other sectors of production and services, as well as predicting the behavior of tourism. For this, the quantitative research method was used as the guiding method, based on the Box - Jenkins methodology and the Holt - Winters exponential smoothing method. It is also possible to characterize tourism management taking into consideration two indicators: cost per weight and average income per tourist, referring to efficiency and effectiveness respectively. In addition, a multivariate analysis of time series was carried out that made it possible to characterize the tourist activity in four fundamental stages in the hotel chain taken as the object of study.

KEY WORDS: Tourist demand; Efficacy; Efficiency; Time series.

\section{INTRODUCCIÓN}

El turismo es uno de los motores mundiales del desarrollo. Cada año se desplazan más personas que nunca antes en la historia. Con una buena planificación y gestión, el turismo puede constituir una fuerza positiva que aporte beneficios a los destinos de todo el mundo, pero si estas son deficientes, puede constituir un factor de degradación (Hącia, 2019). Es considerado como una de las industrias más diversas ya que abarca en la cadena a casi todas las industrias de todos los sectores (Xie et al., 2020).

Por otro lado, el turismo tiene innumerables efectos de orden social, también puede ser visto como una actividad económica por elementos definitorios, tales como la satisfacción de necesidades (ocio y recreación), los gastos y erogaciones que suponen los viajes para los turistas, el consumo y la demanda turística, la generación de riqueza, a través del proceso productivo turístico, entre otros. Es por ello que el sector turístico debe ser capaz de comprender la demanda (Feng et al., 2019).

De ahí la importancia del estudio de la demanda turística de un destino, que se enfoca en conocer las características de los viajeros, relacionadas con el segmento al que pertenecen, el gasto turístico, el nivel de satisfacción de los atractivos del destino, entre otros. Es válido decir que el análisis de los rasgos distintivos de la demanda turística conllevará a diseñar acciones que propendan a mejorar la capacidad del destino para satisfacer las necesidades y deseos de ocio del turista (Wu et al., 2017).

\footnotetext{
“Visión de Futuro" Año 18, Volumen No 25 N 1, Enero - Junio 2021 - Pág 107-132

URL de la Revista: http://visiondefuturo.fce.unam.edu.ar/index.php/visiondefuturo/index

URL del Documento: https://visiondefuturo.fce.unam.edu.ar/index.php/visiondefuturo/issue/view/19

ISSN 1668 - 8708 - Versión en Línea

E-mail: revistacientifica@fce.unam.edu.ar
} 
Por este motivo, la demanda debe pronosticarse para planear el sistema productivo, el abastecimiento y los despachos, de manera que la cadena de suministros opere correctamente. Los pronósticos permiten la obtención de información relevante, precisa y confiable; para lo cual las empresas deben utilizar correctamente los modelos y procedimientos más adecuados para tal fin (C. Li et al., 2020).

A nivel organizacional, el pronóstico de la demanda es un insumo esencial para cualquier decisión en las distintas áreas funcionales: ventas, producción, compras, finanzas y contabilidad. Los pronósticos también son necesarios en los planes de distribución y aprovisionamientos. La importancia de un pronóstico con escaso margen de error es fundamental para la eficiencia y la eficacia. Esto ha sido en gran parte reconocido por diversos autores (S. Li et al., 2018).

Entre los autores más relevante que abordan elementos sobre los estudios de la demanda turística en Cuba se destacan: Madrazo et al. (2009), el cual solo propone un procedimiento para la gestión de la demanda turística y explica que la estimación de la demanda futura es un reto para los estudiosos y planificadores de esta actividad.

Posteriormente Barbosa y Gutiérrez (2010) sugiere la aplicación de los métodos clásicos de descomposición para el análisis de la demanda para conocer entre otros elementos tendencia y estacionalidad sin hacer uso de técnicas avanzadas. En un ámbito más novedoso Gómez (2012), determina la demanda turística con redes neuronales artificiales implicando variables mercadológicas, pero no incluye un análisis de los factores temporales que han afectado la actividad turística como puede ser el efecto de crisis económicas.

En tal sentido, Castro y Fernández (2014) proponen como novedad la inclusión del efecto del cambio climático en el turismo, ese mismo año Cruz-Rodríguez y GonzálezLaucirica (2014), caracterizan el mercado emisor por grupo de edades y sexo aplicando de forma básica la estadística descriptiva. En un contorno más actual Díaz-Pompa et al. (2020) prevé un crecimiento de la demanda turística solo tomando en consideración los datos de la Organización Mundial de Turismo (OMT) del año 2015.

Estos estudios proponen modelos que contemplan como ventaja rasgos del pronóstico de la demanda turística a corto y mediano plazo; pero no abarcan de manera explícita los diversos factores que han influenciado a través del tiempo. Los pronósticos de la demanda turística carecen de proyecciones con distintos márgenes de error para un análisis de mayor profundidad o simplemente mencionan a la previsión de la demanda turística como herramienta fundamental para la toma de decisiones sin hacer uso práctico de la misma.

Dentro de este contexto, la presente investigación tiene como objetivo pronosticar la demanda turística de la Cadena Hotelera Cubanacán en la provincia de Pinar del Río, Cuba

\footnotetext{
"Visión de Futuro" Año 18, Volumen No 25 N 1, Enero - Junio 2021 - Pág 107-132

URL de la Revista: http://visiondefuturo.fce.unam.edu.ar/index.php/visiondefuturo/index

URL del Documento: https://visiondefuturo.fce.unam.edu.ar/index.php/visiondefuturo/issue/view/19

ISSN 1668 - 8708 - Versión en Línea

E-mail: revistacientifica@fce.unam.edu.ar
} 
hasta diciembre del año 2019 y caracterizar la gestión turística tomando en consideración dos indicadores, el costo por peso y los ingresos medios por turista, cada uno haciendo referencia a la eficiencia y eficacia respectivamente.

\section{DESARROLLO}

\section{Marco teórico conceptual}

Los pronósticos de la demanda turística están estrechamente relacionados con la gestión efectiva del destino y la asignación oportuna de recursos, que son de continuo interés para los interesados en el turismo. En tal sentido, diferentes autores han realizado estudios que muestran la diversidad, avance y rigor científico de las herramientas de pronóstico de la demanda en este sector estratégico, entre los primeros se encuentran los de Crouch (1994), el cual realiza una búsqueda exhaustiva de la literatura y donde discute las similitudes y diferencias en los enfoques para los investigadores que deseen realizar estudios similares.

A seguido, Li et al. (2005) propone un estudio sobre modelos y pronósticos de la demanda turística internacional utilizando enfoques econométricos e identifican nuevos trabajos desarrollados y muestra que las aplicaciones de métodos econométricos avanzados mejoran la comprensión de la demanda turística internacional y su encadenamiento con los demás sectores. En este mismo ámbito, Song y Li (2008) realizan una revisión bibliográfica y determinan como hallazgos clave de esta revisión que los métodos utilizados para analizar y pronosticar la demanda del turismo han sido más diversos que los identificados por otros autores previos a su investigación. Además, demuestra que en la literatura han surgido varias técnicas nuevas.

De acuerdo con Song y Li (2008), en lo que respecta a la precisión del pronóstico, el estudio muestra que no existe un modelo único que supere sistemáticamente a otros modelos en todas las situaciones. Además, este estudio identifica nuevas direcciones de investigación, que incluyen mejorar la precisión de la predicción mediante la combinación de predicciones.

En un contexto de mayor actualidad Song et al. (2019) realiza una selección de los autores más relevantes en un ámbito más abarcador que tratan temas sobre el pronóstico de la demanda turística dividiendo estas metodologías en dos grandes grupos: los métodos de series de tiempo y los métodos econométricos, estos asociados a métodos dinámicos y estáticos respectivamente. Asimismo, incluye una nueva clasificación basada en cuatro categorías: series de tiempo, modelos econométricos, métodos basados en inteligencia artificial y métodos basados en juicios.

Dentro de estos estudios se puede determinar que son escazas las investigaciones que

\footnotetext{
"Visión de Futuro" Año 18, Volumen N²5 N 1, Enero - Junio 2021 - Pág 107-132

URL de la Revista: http://visiondefuturo.fce.unam.edu.ar/index.php/visiondefuturo/index

URL del Documento: https://visiondefuturo.fce.unam.edu.ar/index.php/visiondefuturo/issue/view/19

ISSN 1668 - 8708 - Versión en Línea

E-mail: revistacientifica@fce.unam.edu.ar
} 
incluyen análisis multivariado de series temporales incluyendo indicadores de eficiencia y eficacia para el análisis de la gestión turística. La revisión bibliográfica muestra especial atención en los modelos a aplicar. Las metodologías de pronósticos son muy diversas ya que los investigadores emplean tanto series de tiempo como enfoques econométricos para estimar la demanda turística.

\section{Materiales y métodos}

Se utilizaron métodos empíricos de investigación, basados en la observación científica y el análisis documental para caracterizar la situación actual de la demanda turística en Pinar del Río, Cuba.

Entre los métodos estadísticos matemáticos se emplearon herramientas como la metodología Box - Jenkins y el método de alisado exponencial de Holt - Winters. Se manejaron los softwares R 3.6.3 y R Studio 1.2.5033 para el procesamiento de los datos y la información disponible. También fueron utilizados métodos teóricos para reseñar el desarrollo de los actuales procesos de gestión del turismo en la provincia de Pinar del Río, Cuba.

Se usaron como materiales datos mensuales proporcionados por el Mintur que comprenden la cantidad de turistas, el costo por peso y los ingresos medio por turista. El costo por peso se determina mediante la división del costo entre los ingresos (costo/ingresos) mientras, los ingresos medios por turista se establecen dividiendo ingresos entre la cantidad de turistas (ingresos/cantidad de turistas); ambos indicadores se toman como indicadores de eficiencia y eficacia donde a menor costo por peso mayor eficiencia, y a mayor ingresos medios mayor eficacia. El conjunto de datos abarca el periodo entre enero del año 2006 y diciembre del año 2018, para la aplicación de los métodos.

La cadena seleccionada fue Cadena Hotelera Cubanacán, pues la misma es la que genera los mayores ingresos y logra el mayor encadenamiento con el resto de los sectores productivos y de servicios al tener la mayor presencia en el territorio según la información proporcionada por los decisores.

\section{Metodología Box - Jenkins}

En este estudio, uno de los procedimientos estadísticos que se implementa para las predicciones es el que se muestra en la figura 1. Las predicciones comienzan con la identificación del Proceso Autorregresivo de Integración de Medias Móviles utilizando las funciones de auto - arima de R. Con los parámetros estimados se conforma el modelo, que se valida mediante el análisis de los residuos. Si este modelo es adecuado se procede al pronóstico. Los residuos deben de estar incorrelacionados $\left(\rho_{\mathrm{k}} \approx 0\right.$, donde $\rho_{\mathrm{k}}=$ "Visión de Futuro" Año 18, Volumen $N^{\circ} 25$ N 1, Enero - Junio 2021 - Pág 107-132 URL de la Revista: http://visiondefuturo.fce.unam.edu.ar/index.php/visiondefuturo/index URL del Documento: https://visiondefuturo.fce.unam.edu.ar/index.php/visiondefuturo/issue/view/19 
$\left.\frac{\operatorname{cov}\left(y_{t}, y_{t-k}\right)}{\sqrt{\operatorname{var}\left(y_{t}\right) \operatorname{var}\left(y_{t-k}\right)}}\right)$ para que el modelo sea adecuado (Bakar \& Rosbi, 2017). Esto es lo que se conoce como metodología Box - Jenkins.

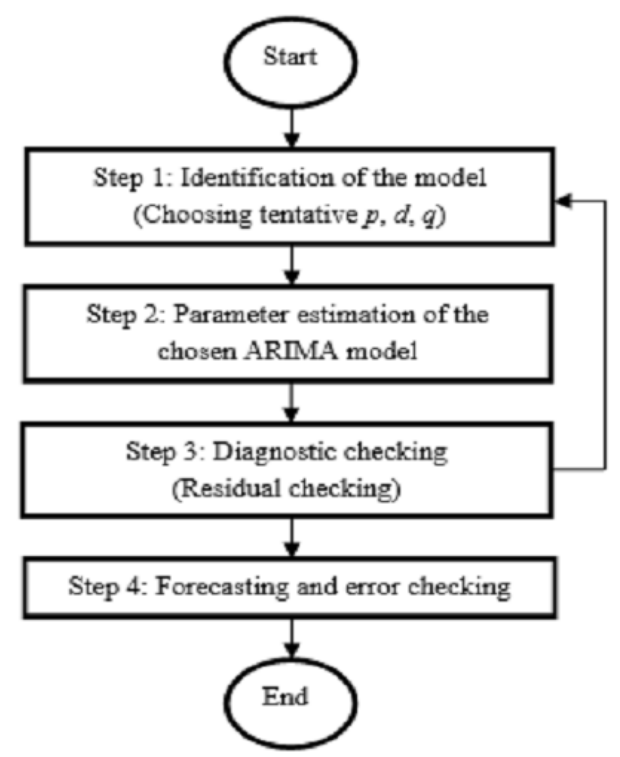

Figura № 1: Resumen del procedimiento para la estadística de predicción Fuente: Bakar y Rosbi (2017)

\section{Modelo Auto Regresivo Integrado de Media Móvil}

El modelo Autorregresivo Integrado de Media Móvil (ARIMA) está compuesto por dos modelos, el Autorregresivo (AR) y el de Medias Móviles (MA). El modelo ARIMA tiene parámetros específicos para las series de tiempo: los parámetros p y q, los cuales representan el orden del AR y el orden de la MA, respectivamente. Se adiciona un parámetro d que representa el número de las diferencias (Bakar \& Rosbi, 2017).

Conocida como metodología de Box - Jenkins (BJ), pero técnicamente conocida como metodología ARIMA (Al-Musaylh et al., 2018), marcó el comienzo de una nueva generación de herramientas de pronóstico.

El modelo AR se escribe como: $y_{t}=c+a_{1} y_{t-1}+\cdots+a_{p} y_{t-p}+u_{t}$, dónde $a_{1}, a_{2}, a_{3}, \ldots, a_{p}$ son los parámetros del $A R$, c es una constante, $p$ es el orden del AR, y $u_{t}$ es el ruido blanco. El modelo MA se puede escribir como: $y_{t}=\mu+u_{t}+m_{1} u_{t-1}+\cdots+m_{q} u_{t-q}$, dónde $\mathrm{m}_{1}, \mathrm{~m}_{2}, \mathrm{~m}_{3}, \ldots, \mathrm{m}_{\mathrm{q}}$ son los parámetros de $M A, u_{\mathrm{t}}, \mathrm{u}_{\mathrm{t}-1}, \ldots, \mathrm{u}_{\mathrm{t}-\mathrm{q}}$ son los términos del ruido blanco y $\mu$ es lo que se espera de $y_{t}$. Integrando estos modelos se obtiene el modelo ARIMA que tiene la expresión siguiente: $\mathrm{y}_{\mathrm{t}}=\mathrm{c}+\mathrm{a}_{1} \mathrm{y}_{\mathrm{t}-1}+\cdots+\mathrm{a}_{\mathrm{p}} \mathrm{y}_{\mathrm{t}-\mathrm{p}}+\mathrm{u}_{\mathrm{t}}+\mu+\mathrm{u}_{\mathrm{t}}+$ $\mathrm{m}_{1} \mathrm{u}_{\mathrm{t}-1}+\cdots+\mathrm{m}_{\mathrm{q}} \mathrm{u}_{\mathrm{t}-\mathrm{q}}$, dónde $\mathrm{p}$ y $\mathrm{q}$ son los términos del proceso autorregresivo y de medias 
móviles respectivamente.

\section{Modelo Auto Regresivo Integrado de Media Móvil Estacional}

El Proceso Autorregresivo Integrado de Media Móvil Estacional (SARIMA) es una extensión del ARIMA en el caso de que la serie estacionaria presente la componente estacional, la cual incluye nuevos términos para la diferenciación de orden 12 (Bakar \& Rosbi, 2017). Los modelos ARIMA estacionales (P, D, Q) complementan el modelo general no estacional ARIMA ( $p, d, q)$, desarrollados para captar los patrones estacionales trimestrales o semestrales presentes en las series temporales (Box et al., 1970). La combinación de modelos ARIMA ( $p, d, q)$ no estacionales con ARIMA (P, D, Q) estacionales conduce al modelo SARIMA (p, d , q) × (P, D , Q), también conocido como ARIMA multiplicativo (Milenković et al., 2018).

En forma agregada, su representación general es: $(1-\mathrm{B})^{\mathrm{d}}\left(1-\mathrm{B}^{\mathrm{s}}\right)^{\mathrm{D}} \mathrm{y}_{\mathrm{t}}=\alpha+$ $\frac{\left(1-\theta_{1} B-\cdots-\theta_{q} B^{q}\right)\left(1-\Theta_{1} B^{s}-\cdots-\Theta_{Q} B^{S Q}\right)}{\left(1-\phi_{1} B-\cdots-\phi_{p} B^{p}\right)\left(1-\Phi_{1} B^{s}-\cdots-\Phi_{P} B^{S P}\right)} \varepsilon_{t}$, donde: $d$ es el número de diferencias regulares, $D$ es el número de diferencias estacionales, $s$ es la amplitud estacional, $\alpha$ constante óptima, q es el número de componentes de medias móviles, $Q$ es el número de componentes de medias móviles estacionales, $\theta_{\mathrm{q}}$ son los coeficientes de medias móviles, $\Theta_{\mathrm{Q}}$ son los coeficientes de medias móviles estacionales, $\mathrm{p}$ es el número de componentes autorregresivos, $\mathrm{P}$ es el número de componentes autorregresivos estacionales, $\emptyset_{\mathrm{p}}$ son coeficientes de los procesos autorregresivos, $\Phi_{\mathrm{p}}$ son los coeficientes de los procesos autorregresivos estacionales

\section{Función de autocorrelación}

La función de autocorrelación (ACF) es una herramienta de gran utilidad en la identificación del orden de un modelo de MA. La ACF de un MA(q) se anula después del retardo $\mathrm{q}$, es decir, $\rho_{\mathrm{k}} \approx 0$, para $\mathrm{k}>\mathrm{q}$, entonces se tiene que el proceso puede ser modelizado mediante un proceso de medias móviles de orden q, MA(q) (Rahman \& Ahmar, 2017). La ACF representa gráficamente los valores de correlación para k retardos de tiempo (Petrevska, 2017).

Dado el supuesto de serie estacionaria, donde se tiene $\operatorname{var}\left(\mathrm{y}_{\mathrm{t}}\right)=\operatorname{var}\left(\mathrm{y}_{\mathrm{t}-1}\right)$ la función de autocorrelación se le denomina función de autocorrelación parcial (PACF), la cual representa los valores para un desfase $\mathrm{k}$ y se implementa para seleccionar el orden del

\footnotetext{
"Visión de Futuro" Año 18, Volumen N²5 N 1, Enero - Junio 2021 - Pág 107-132

URL de la Revista: http://visiondefuturo.fce.unam.edu.ar/index.php/visiondefuturo/index

URL del Documento: https://visiondefuturo.fce.unam.edu.ar/index.php/visiondefuturo/issue/view/19

ISSN 1668 - 8708 - Versión en Línea

E-mail: revistacientifica@fce.unam.edu.ar
} 
proceso AR. Esta PACF se construye a partir de la expresión siguiente: $\rho_{\mathrm{k}}=\frac{\operatorname{cov}\left(\mathrm{y}_{\mathrm{t}}, \mathrm{y}_{\mathrm{t}-\mathrm{k}}\right)}{\operatorname{var}\left(\mathrm{y}_{\mathrm{t}}\right)}$. Ambas se utilizan para el análisis de los residuos y para comprobar si el modelo es adecuado o no (Milenković et al., 2018).

\section{Alisado exponencial de Holt - Winters}

Además de la metodología Box - Jenkins, se implementa el modelo Holt - Winters. El mismo incorpora un conjunto de procedimientos que conforman el núcleo de la familia de series temporales de suavizamiento o alisamiento exponencial propuestos a finales de la década de 1950 (Brown, 1959; Holt, 1957; Winters, 1960).

A diferencia de muchas otras técnicas, el modelo Holt - Winters puede adaptarse fácilmente a cambios y tendencias, así como a patrones estacionales.

Holt - Winters se utiliza habitualmente por muchas compañías para pronosticar la demanda a corto plazo cuando los datos de venta contienen tendencias y patrones estacionales de un modo subyacente.

La estructura básica de la técnica Holt - Winters se basa en la modificación de la anterior técnica de suavizamiento exponencial, la cual se amplía para incluir un parámetro de ajuste estacional. En este trabajo se considera el suavizado de Winters que es un método de suavizado de series temporales que presentan tendencia y estacionalidad, la misma consiste en tres ecuaciones, cada una de las cuales actualiza un factor asociado con cada uno de los componentes de la serie: nivel aleatoriedad, tendencia y estacionalidad.

El método utiliza un conjunto de estimaciones recursivas a partir de la serie histórica. Estas estimaciones utilizan constantes $\alpha ; \beta$ y $\gamma$ para la actualización del nivel, la tendencia y la estacionalidad respectivamente en referencia a las componentes de las series temporales en cuestión (Su et al., 2018).

Existen dos variaciones de este método: El método aditivo cuando las variaciones estacionales son aproximadamente constante a lo largo de la serie, con la siguiente forma del

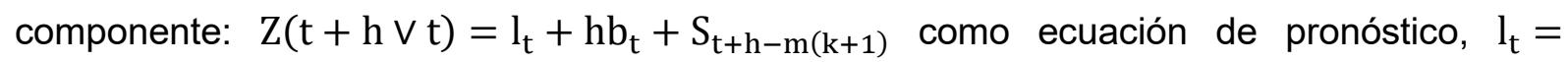
$\alpha\left(y_{t}-S_{t-m}\right)+(1-\alpha)\left(l_{t-1}\right) b_{t-1}$ como ecuación de nivel, $b_{t}=\beta\left(l_{t}+l_{t-1}\right)+(1-\beta) b_{t-1}$ representando la tendencia y $S_{t}=\gamma\left(y_{t}-l_{t-1}+b_{t-1}\right)+(1-\gamma) S_{t-1}$ como ecuación que representa la estacionalidad.

El método multiplicativo se aplica cuando las variaciones estacionales cambian proporcionalmente al nivel de la serie, con la siguiente forma del componente: $\mathrm{Z}(\mathrm{t}+\mathrm{h} \vee \mathrm{t})=$ (l) $\left.\mid \mathrm{t}+\mathrm{h} \mathrm{b}_{\mathrm{t}}\right) * \mathrm{~S}_{\mathrm{t}+\mathrm{h}-\mathrm{m}(\mathrm{k}+1)}$ ecuación de pronóstico, $\mathrm{l}_{\mathrm{t}}=\alpha\left(\frac{\mathrm{y}_{\mathrm{t}}}{\mathrm{s}_{\mathrm{t}-\mathrm{m}}}\right)+(1-\alpha)\left(\mathrm{l}_{\mathrm{t}-1}\right) \mathrm{b}_{\mathrm{t}-1}$

\footnotetext{
"Visión de Futuro" Año 18, Volumen N²5 N 1, Enero - Junio 2021 - Pág 107-132

URL de la Revista: http://visiondefuturo.fce.unam.edu.ar/index.php/visiondefuturo/index

URL del Documento: https://visiondefuturo.fce.unam.edu.ar/index.php/visiondefuturo/issue/view/19

ISSN 1668 - 8708 - Versión en Línea

E-mail: revistacientifica@fce.unam.edu.ar
} 
ecuación de nivel, $b_{t}=\beta\left(l_{t}+l_{t-1}\right)+(1-\beta) b_{t-1}$ ecuación de tendencia, $S_{t}=$ $\gamma\left(\frac{\mathrm{y}_{\mathrm{t}}}{\mathrm{l}_{\mathrm{t}-1}+\mathrm{b}_{\mathrm{t}-1}}\right)+(1-\gamma) \mathrm{S}_{\mathrm{t}-1}$ ecuación de estacionalidad.

\section{Resultados}

Con el fin de analizar el comportamiento de la serie demanda turística en la Cadena Hotelera Cubanacán, en el período comprendido entre enero del 2006 y diciembre del 2018, se procede a realizar la representación gráfica de la misma. En la figura 2, se puede clasificar la serie como estacionaria, pues oscila alrededor de un valor medio.

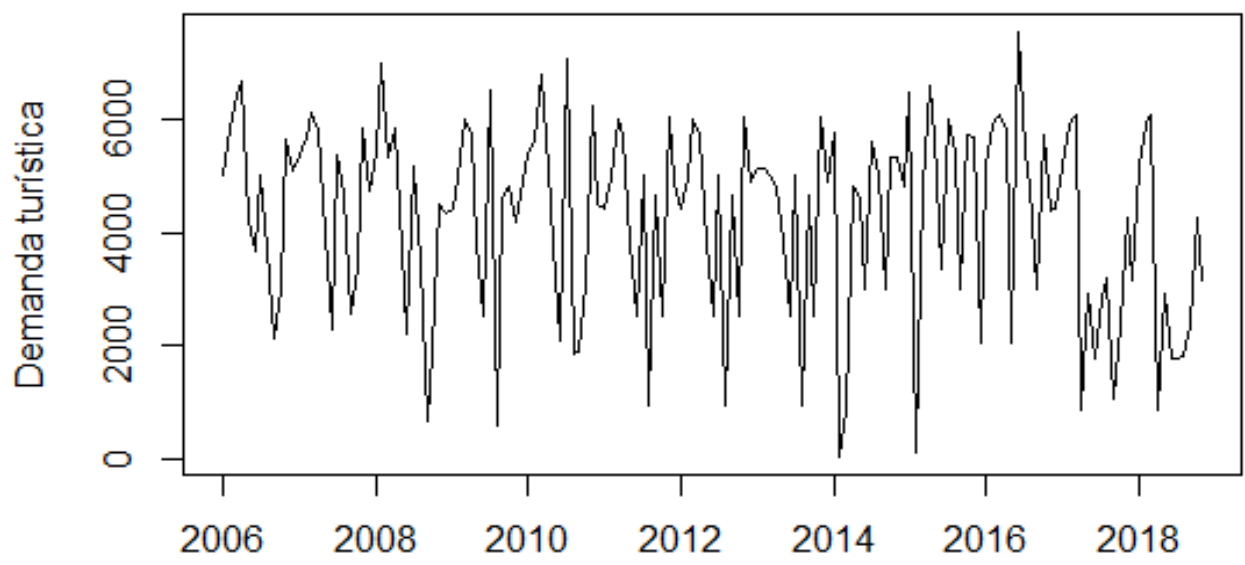

Figura $N^{\circ}$ 2: Serie temporal de la demanda turística mensual (Cadena Hotelera Cubanacán, 2006-2018) Fuente: R, versión 3.6.3

Al descomponer la serie temporal para el análisis de la tendencia y la estacionalidad, se presenta un gráfico que contiene cada componente de la serie, obtenido mediante el método de las medias móviles facilitado por el asistente matemático R. En la figura 3 se muestran los valores observados, la componente estacional, la tendencia y los residuos. Es apreciable que la demanda turística tiende a disminuir en el último año y la componente más fuerte es la estacionalidad.

Se observan además tres fluctuaciones importantes: la primera comienza en el año 2008 a causa de la crisis económica mundial y el azote de los huracanes Gustav e lke; la segunda se evidencia a partir del año 2013 al entrar en pánico el mundo por el brote pandémico de la enfermedad del Ébola y la tercera posterior al año 2015, debido a la apertura de las relaciones Cuba-Estados Unidos; no obstante, mostrando un decrecimiento a partir del año 2017 como consecuencia del retroceso de estas relaciones. 


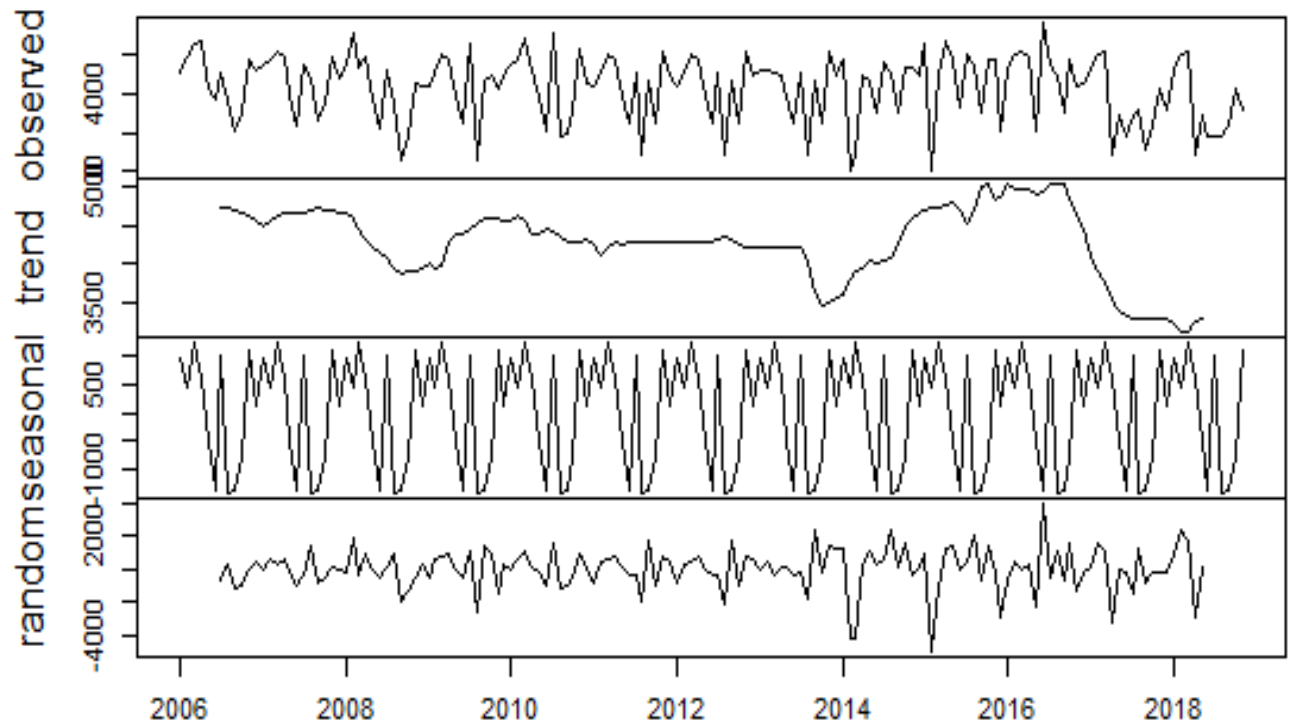

Figura №3: Método de descomposición por medias móviles modelo aditivo para la serie temporal demanda turística en la Cadena Hotelera Cubanacán Fuente: R, versión 3.6.3

En los gráficos de cajas, se puede apreciar que los años de mayores picos fueron 2006, 2007, 2013 y 2016. En el año 2013 ocurrió la mayor cantidad de visitantes turistas con una baja variabilidad y se corrobora la disminución a partir del año 2017 (figura 4a).
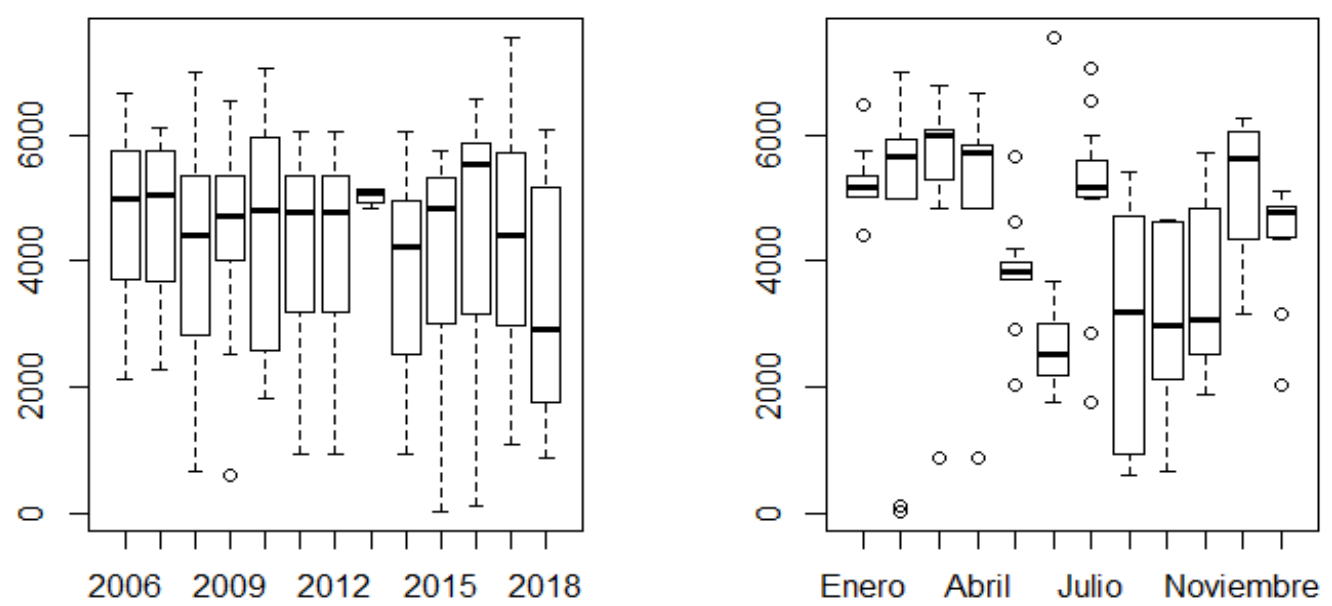

Figura No 4 (a y b): Gráficos de cajas de la demanda turística por año y por mes (Cadena Hotelera Cubanacán)

Fuente: $\mathrm{R}$, versión 3.6 .3

Si se describe la serie por meses se pueden observar las temporadas que predominan en la cadena hotelera: temporada alta y temporada baja, correspondientes a los meses de invierno y verano. Se extrae del gráfico de la figura $4 \mathrm{~b}$ que los meses de más estabilidad turística son enero y mayo. No ocurriendo así para los meses desde agosto hasta noviembre como muestra la tabla 1.

\footnotetext{
"Visión de Futuro" Año 18, Volumen N²5 N 1, Enero - Junio 2021 - Pág 107-132

URL de la Revista: http://visiondefuturo.fce.unam.edu.ar/index.php/visiondefuturo/index

URL del Documento: https://visiondefuturo.fce.unam.edu.ar/index.php/visiondefuturo/issue/view/19

ISSN 1668 - 8708 - Versión en Línea

E-mail: revistacientifica@fce.unam.edu.ar
} 
Tabla $N^{\circ}$ 1: Media para la demanda turística por años y por meses

\begin{tabular}{cccccc}
\hline & Media & Año & Media & Año & Media \\
\hline Ene & 5171 & 2006 & 4699 & 2018 & 3456 \\
\hline Feb & 4779 & 2007 & 4644 & & \\
\hline Mar & 5506 & 2008 & 4121 & & \\
\hline Abr & 4950 & 2009 & 4419 & & \\
\hline May & 3778 & 2010 & 4448 & & \\
\hline Jun & 2902 & 2011 & 4287 & \\
\hline Jul & 5061 & 2012 & 4287 & \\
\hline Ago & 2870 & 2013 & 5023 & & \\
\hline Sep & 2936 & 2014 & 3798 & & \\
\hline Oct & 3616 & 2015 & 4013 & & \\
\hline Nov & 5177 & 2016 & 4566 & & \\
\hline Dic & 4383 & 2017 & 4292 & & \\
\hline & & Fuente: Elaboración propia &
\end{tabular}

Anteriormente, se identificó que la serie demanda turística en la Cadena Hotelera Cubanacán es estacionaria y cuya componente más fuerte es la estacional, como muestra el correlograma de la figura 5 al contener máximos relativos con período 12.

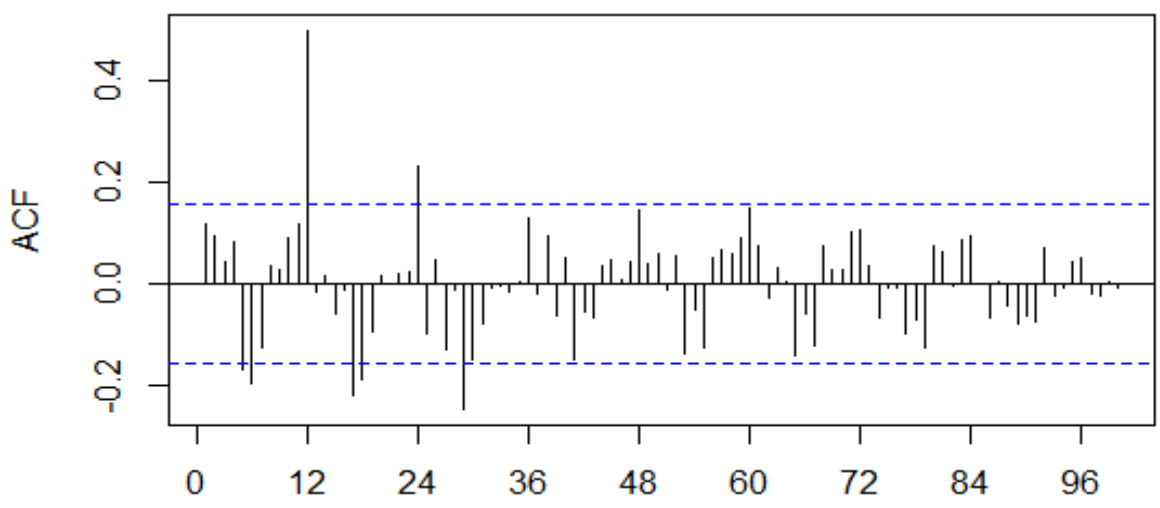

Figura № 5: Gráfica de la función de autocorrelación para la serie temporal demanda turística en la Cadena Hotelera Cubanacán Fuente: R, versión 3.6.3

Teniendo en cuenta las características de la serie, Jadhav et al. (2017) y Petrevska (2017) plantean que la opción más recomendable es la utilización de los modelos SARIMA. Será necesario realizar una diferenciación de orden 12 para eliminar la estacionalidad y de este modo lograr una serie puramente estacionaria previa a la aplicación del modelo.

La función auto - arima de $\mathrm{R}$, selecciona el mejor modelo de manera automática esto "Visión de Futuro" Año 18, Volumen $N^{\circ} 25$ No 1, Enero - Junio 2021 - Pág 107-132 URL de la Revista: http://visiondefuturo.fce.unam.edu.ar/index.php/visiondefuturo/index URL del Documento: https://visiondefuturo.fce.unam.edu.ar/index.php/visiondefuturo/issue/view/19 ISSN 1668 - 8708 - Versión en Línea 
se corrobora en el trabajo de Liu et al. (2020), los resultados que se obtienen a través del software se muestran en la tabla 2.

Tabla No 2: Modelos SARIMA para la serie temporal demanda turística

\begin{tabular}{ccc}
\hline \multicolumn{3}{c}{ Serie demanda turística ARIMA (0.0.0) (1.0.0) [12] } \\
\hline Coeficientes & Sar1 & Media \\
s. e & 0.5412 & 4217.399 \\
AIC $=2697.39$ & 0.0683 & 226.816 \\
MPE $=-138.9312$ & AlCc $=2697.55$ & BIC $=2706.52$ \\
& MAPE $=157.0537$ & MASE $=1.064214$ \\
\hline
\end{tabular}

Fuente: Elaboración propia

El modelo más adecuado que minimiza todas las medidas de dispersión es un modelo con una diferenciación de orden doce y de orden uno en la parte autorregresiva de la estacionalidad o sea un modelo $\operatorname{SARIMA}(0,0,0)(1,0,0)$.

Para validar el modelo se realiza el contraste de Ljung-Box-Pierce, también conocido como test de portmanteau. La hipótesis nula es que las primeras autocorrelaciones son nulas. El resultado que se tiene en la tabla 3 implica que las correlaciones son iguales a cero y, por tanto, se puede asumir que los residuos se comportan como ruido blanco.

Tabla No 3: Prueba de Ljung-Box

Box-Ljung test data: residuals

X-squared $=1.7221, \mathrm{df}=1, \mathrm{p}$-value $=0.1894$

Fuente: Elaboración propia

La representación gráfica de los residuos estandarizados de la figura 6 muestra que estos varían en torno a cero, sin tendencia, con varianza constante y no hay valores atípicos. Aproximadamente el $95 \%$ de los residuos estandarizados deben de estar entre -2 y 2 desviaciones típicas.

\footnotetext{
"Visión de Futuro" Año 18, Volumen N²5 N 1, Enero - Junio 2021 - Pág 107-132

URL de la Revista: http://visiondefuturo.fce.unam.edu.ar/index.php/visiondefuturo/index

URL del Documento: https://visiondefuturo.fce.unam.edu.ar/index.php/visiondefuturo/issue/view/19

ISSN 1668 - 8708 - Versión en Línea

E-mail: revistacientifica@fce.unam.edu.ar
} 
Standardized Residuals

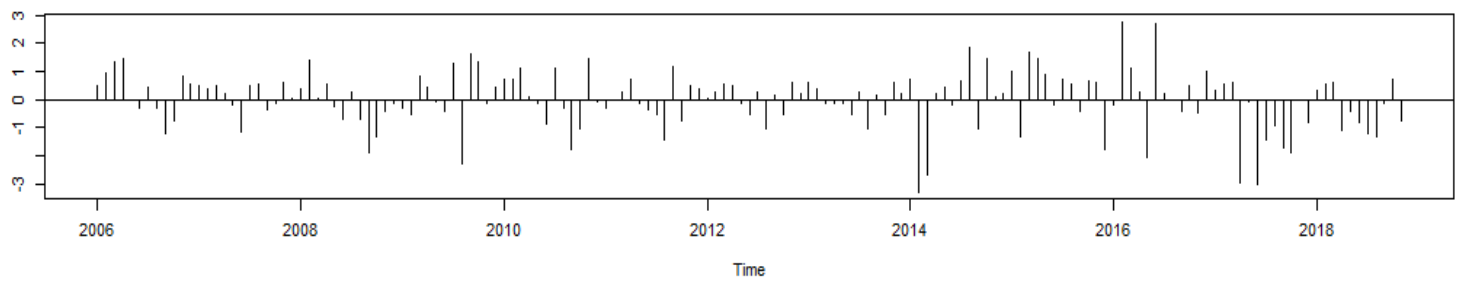

ACF of Residuals

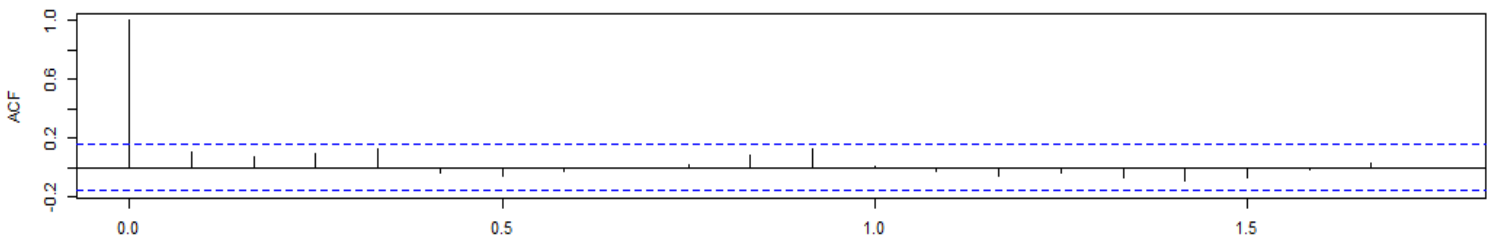

Lag

p values for Ljung-Box statistic

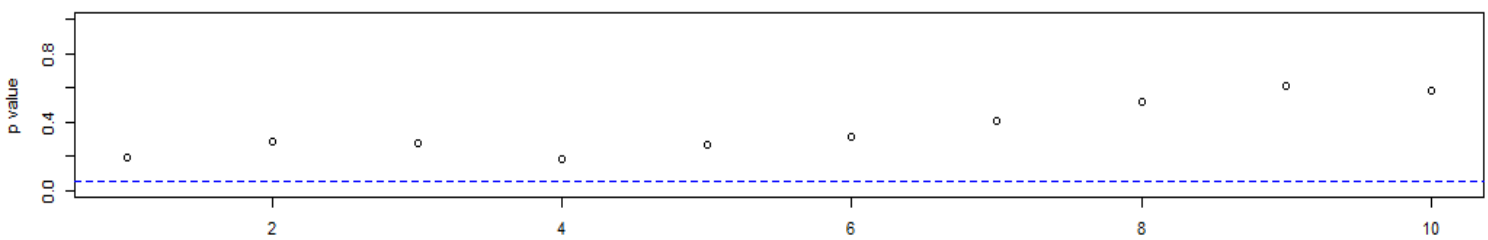

Figura $N^{\circ}$ 6. Gráfica de los residuos estandarizados del modelo SARIMA

Fuente: R, versión 3.6.3

El pronóstico de la demanda turística mensual de la Cadena Hotelera Cubanacán para el año 2019 se muestra en la salida de $\mathrm{R}$ de la tabla 4. En esta se observa el pronóstico a través de intervalos para un 80 y un $95 \%$ de confianza.

Tabla N 4: Pronóstico de la demanda turística para el año 2019

\begin{tabular}{ccccccc}
\hline & Año & Pronóstico & Inf 80 & Sup 80 & Inf 95 & Sup 95 \\
\hline Ene & 2019 & 4740.0 & 2924.5 & 6555. & 1963.4 & 7516.5 \\
\hline Feb & 2019 & 5141.6 & 3326.1 & 6957.0 & 2365.0 & 7918.1 \\
\hline Mar & 2019 & 5232.5 & 3417.0 & 7048.0 & 2456.0 & 8009.0 \\
\hline Abr & 2019 & 2406.1 & 590.7 & 4221.6 & -370.3 & 5182.7 \\
\hline May & 2019 & 3515.7 & 1700.2 & 5331.2 & 739.2 & 6292.2 \\
\hline Jun & 2019 & 2880.8 & 1065.3 & 4696.3 & 104.3 & 5657.3 \\
\hline Jul & 2019 & 2880.8 & 1065.3 & 4696.3 & 104.3 & 5657.3 \\
\hline Ago & 2019 & 2902.5 & 1087.0 & 4717.9 & 125.9 & 5679.0 \\
\hline Sep & 2019 & 3203.4 & 1387.9 & 5018.9 & 426.9 & 5979.9 \\
\hline Oct & 2019 & 4230.7 & 2415.2 & 6046.1 & 1454.1 & 7007.2 \\
\hline Nov & 2019 & 3644.0 & 1828.5 & 5459.4 & 867.4 & 6420.5 \\
\hline Dic & 2019 & 3907.0 & 1842.7 & 5971.3 & 749.9 & 7064.1 \\
\hline
\end{tabular}

Fuente: Elaboración propia

“Visión de Futuro" Año 18, Volumen No 25 No 1, Enero - Junio 2021 - Pág 107-132

URL de la Revista: http://visiondefuturo.fce.unam.edu.ar/index.php/visiondefuturo/index

URL del Documento: https://visiondefuturo.fce.unam.edu.ar/index.php/visiondefuturo/issue/view/19

ISSN 1668 - 8708 - Versión en Línea

E-mail: revistacientifica@fce.unam.edu.ar 
Mediante la función plot de $\mathrm{R}$ se puede obtener la representación gráfica de la serie demanda turística, con su pronóstico para el próximo año como se muestra en la figura 7.

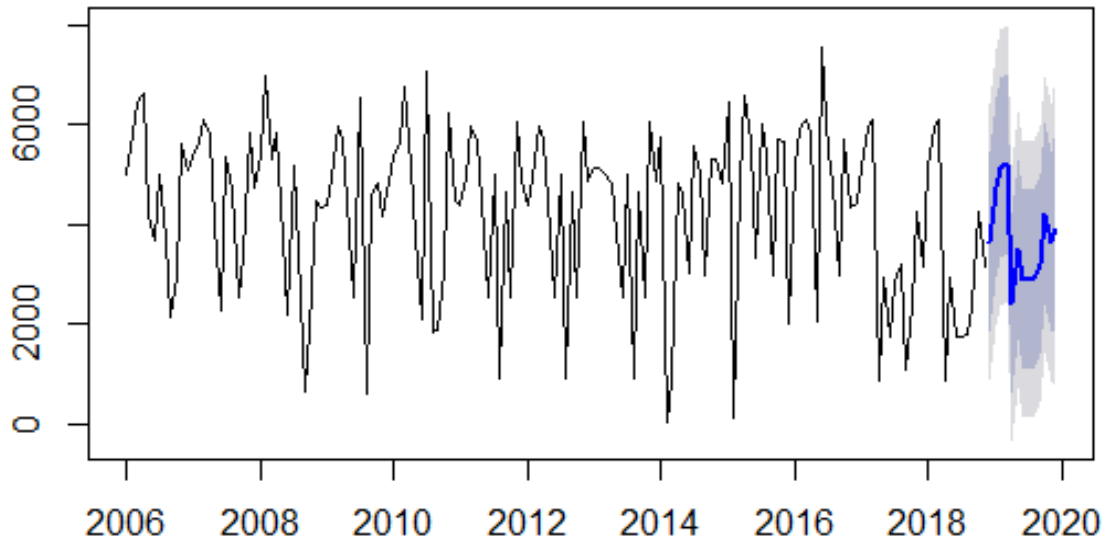

Figura № 7: Pronóstico de la demanda turística en la Cadena Hotelera Cubanacán para el año 2019 Fuente: R, versión 3.6.3

No basta solo con analizar el pronóstico de la demanda en un determinado sector, también es necesario conocer el comportamiento de su eficiencia y eficacia, en este caso visto desde dos indicadores: costo por peso e ingresos promedio por turista para apoyar a la serie demanda turística y obtener diferentes pronósticos que ayuden al Mintur y a la Cadena Hotelera Cubanacán a entender mejor cuál es el comportamiento de esta última y por qué.

En tal sentido, se efectúa el análisis de la Cadena Hotelera Cubanacán a través del indicador de eficiencia definido previamente, y se realiza la representación gráfica para la serie costo por peso desde el año 2006 hasta el año 2018 como se muestra en la figura 8.

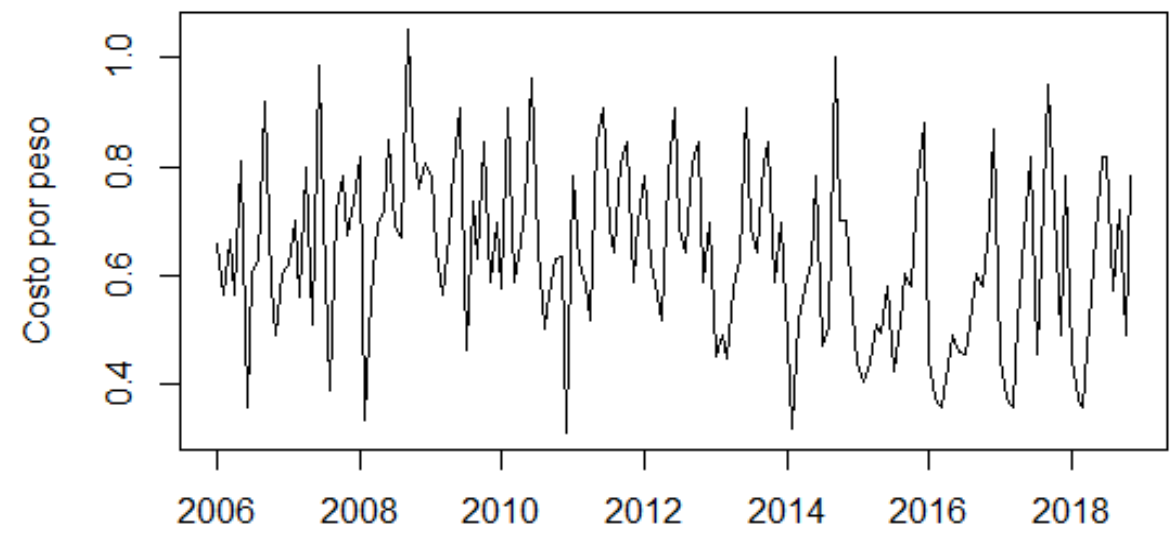

Figura No 8: Serie temporal del costo por peso mensual (Cadena Hotelera Cubanacán, 2006-2018) Fuente: R, versión 3.6.3

Las características que muestra la serie costo por peso provocan que el método de descomposición utilizado sea el ajuste por regresión polinómica local (Loess por sus siglas en inglés).

En la figura 9 se puede comprobar que la componente que predomina es la

\footnotetext{
"Visión de Futuro" Año 18, Volumen N²5 N 1, Enero - Junio 2021 - Pág 107-132

URL de la Revista: http://visiondefuturo.fce.unam.edu.ar/index.php/visiondefuturo/index

URL del Documento: https://visiondefuturo.fce.unam.edu.ar/index.php/visiondefuturo/issue/view/19

ISSN 1668 - 8708 - Versión en Línea

E-mail: revistacientifica@fce.unam.edu.ar
} 
estacionalidad. La gráfica de la serie suavizada (tendencia) evidencia la influencia de la crisis económica mundial y el paso de los huracanes Gustav e Ike en el 2008 con un aumento considerable del costo por peso. A partir del año 2015 existe un decrecimiento de la serie producto a la ocurrencia del cambio de política de Estados Unidos hacia Cuba, manifestándose de modo inverso a partir del año 2017.

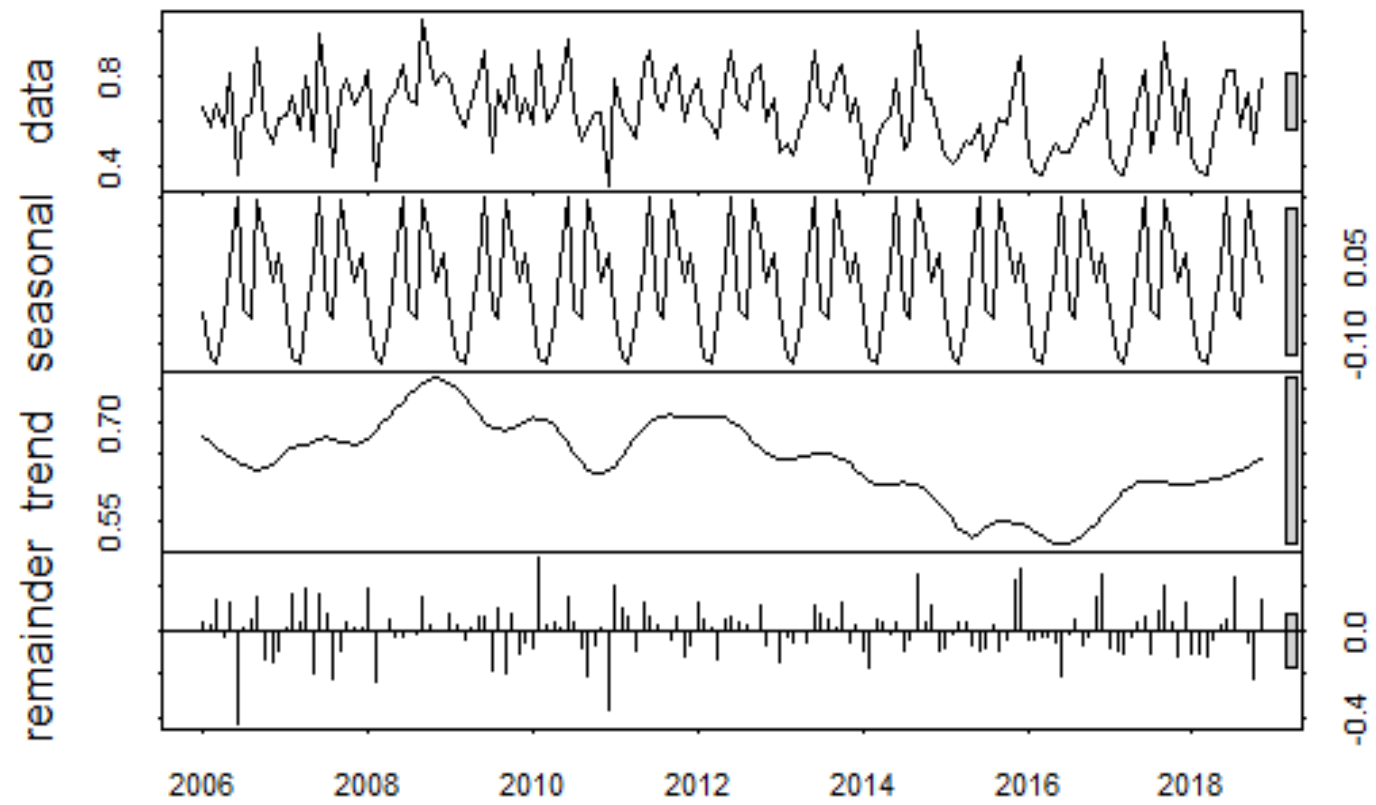

Figura $N^{\circ}$ 9: Método de descomposición por Loess modelo aditivo de la serie temporal costo por peso mensual

Fuente: R, versión 3.6.3

El correlograma de la serie, figura 10, permite comprobar que existe un predominio de la componente estacional, evidenciado por la presencia de un máximo relativo para el retardo 12; además de estar presente la componente tendencia, pero en menor grado al ir los valores de la función de autocorrelación de positivos a negativos.

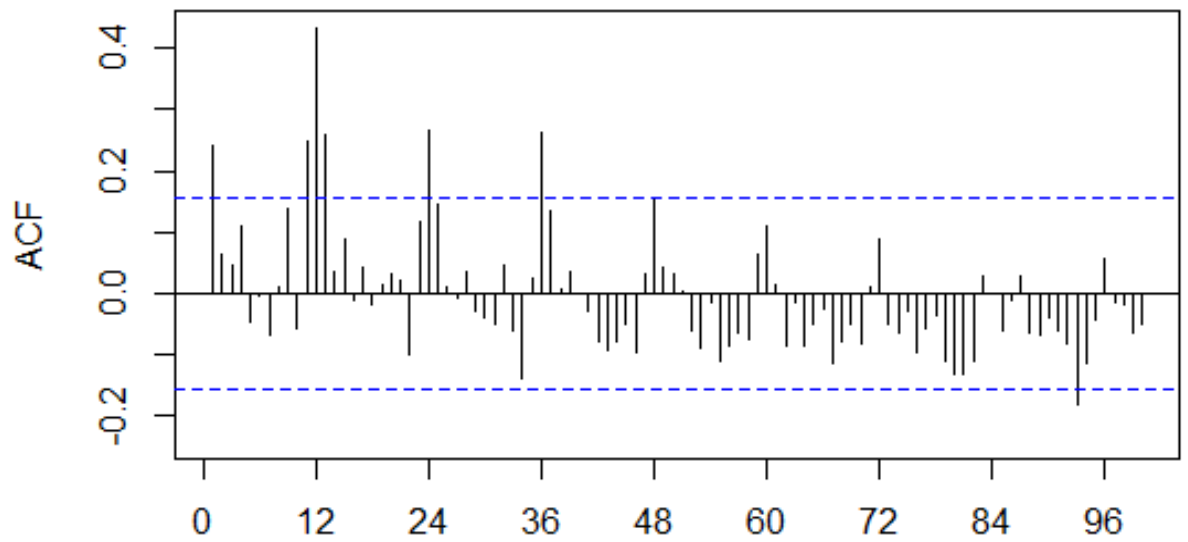

Figura N ${ }^{\circ}$ 10: Gráfica de la función de autocorrelación de la serie temporal costo por peso Fuente: R, versión 3.6.3

\footnotetext{
"Visión de Futuro" Año 18, Volumen N²5 N 1, Enero - Junio 2021 - Pág 107-132

URL de la Revista: http://visiondefuturo.fce.unam.edu.ar/index.php/visiondefuturo/index

URL del Documento: https://visiondefuturo.fce.unam.edu.ar/index.php/visiondefuturo/issue/view/19

ISSN 1668 - 8708 - Versión en Línea

E-mail: revistacientifica@fce.unam.edu.ar
} 
A partir de las características de la serie costo por peso, se usó como herramienta de pronóstico el Alisado de Holt - Winters, pues ésta se emplea con series que presentan tendencia y estacionalidad. Como bien explica Castillo (2017) al determinar que en series cronológicas con este tipo de característica los alisados exponenciales minimiza las medidas referentes al error. La herramienta depende de los parámetros alpha, beta y gamma. Este método entrega las medidas de dispersión para cada modelo obtenido, quedando seleccionado aquel que presente los valores mínimos de variabilidad como se tiene en la tabla 5 , lograda a partir de la salida del software.

Tabla No 5: Holt-Winters exponencial

\begin{tabular}{cccccrr}
\hline \multicolumn{7}{c}{ Coeficientes } \\
\hline Alpha & Beta & Gamma & $\mathrm{a}$ & $\mathrm{b}$ & $\mathrm{s} 1$ & $\mathrm{~s} 2$ \\
\hline 0.072731 & 0.054055 & 0.369321 & 0.641201 & 0.00134 & 0.14925 & -0.15682 \\
\hline $\mathrm{s} 3$ & $\mathrm{~s} 4$ & $\mathrm{~s} 5$ & $\mathrm{~s} 6$ & $\mathrm{~s} 7$ & $\mathrm{~s} 8$ & $\mathrm{~s} 9$ \\
\hline-0.22486 & -0.224 & -0.10303 & 0.011468 & 0.129945 & -0.01836 & -0.05278 \\
\hline s10 & $\mathrm{s} 11$ & $\mathrm{~s} 12$ & & & \\
\hline 0.140653 & -0.01305 & 0.051973 & & & \\
\hline ME & RMSE & MAE & MPE & MAPE & MASE \\
\hline-0.00632 & 0.141682 & 0.10657 & -4.8446 & 17.97706 & 0.920366 \\
\hline
\end{tabular}

Fuente: Elaboración propia

El modelo estimado mediante la función Holt-Winters automática de $\mathrm{R}$, que minimiza la de dispersión, es el que comprende los parámetros con valores de $0.0727,0.054$ y 0.3693 para alpha, beta y gamma respectivamente. Se corrobora que en este modelo la componente de mayor peso para la predicción es la estacionalidad (gamma $=0.3693$ ).

La calidad del modelo para el pronóstico de la serie cronológica costo por peso se verifica mediante el análisis de los residuos a través de la prueba de contraste de Ljung-BoxPierce. La aplicación de esta prueba permite aseverar que los residuos se comportan como ruido blanco como se muestra en la tabla 6 , según el valor de probabilidad ( $p$ valor $>0.05$ )

Tabla N 6: Prueba de Ljung-Box

Box-Ljung test data: residuals

X-squared $=1.2783, \mathrm{df}=1, \mathrm{p}$-value $=0.2582$

Fuente: Elaboración propia

La representación gráfica de los residuos confirma el cumplimiento de los supuestos básicos, ésta permite afirmar que el modelo seleccionado para la predicción posee la calidad

\footnotetext{
“Visión de Futuro" Año 18, Volumen No 25 No 1, Enero - Junio 2021 - Pág 107-132

URL de la Revista: http://visiondefuturo.fce.unam.edu.ar/index.php/visiondefuturo/index

URL del Documento: https://visiondefuturo.fce.unam.edu.ar/index.php/visiondefuturo/issue/view/19

ISSN 1668 - 8708 - Versión en Línea

E-mail: revistacientifica@fce.unam.edu.ar
} 
adecuada como se muestra en la figura 11.

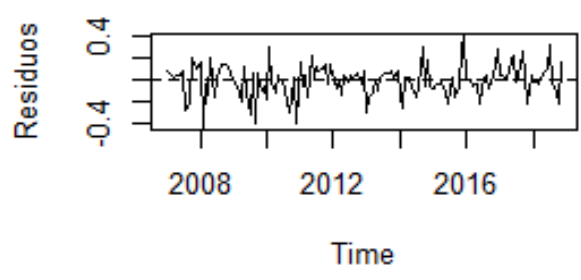

Series Residuos

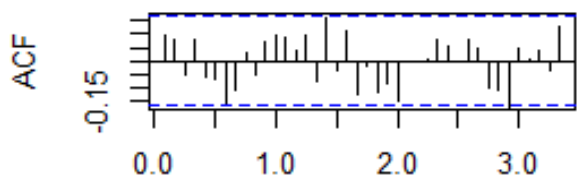

Laq

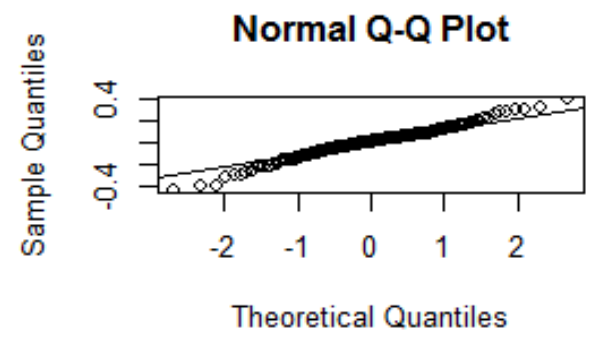

Histogram of Residuos

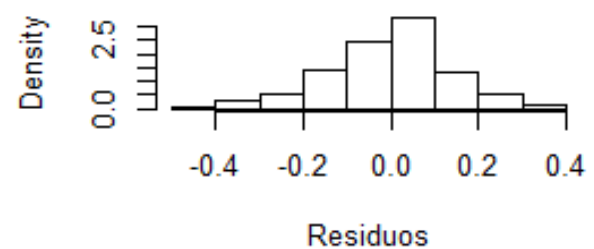

Figura N ${ }^{\circ}$ 11: Gráfica de los residuos estandarizados del modelo Holt-Winters Fuente: R, versión 3.6.3

A partir de la librería forecast de $\mathrm{R}$ se conforma la predicción para el año 2019 , así como la estimación por intervalos para una confiabilidad del 80 y el $95 \%$. Los resultados se muestran en la tabla siguiente.

Tabla N 7: Pronóstico de la demanda turística para el año 2019

\begin{tabular}{ccccccc}
\hline & Año & Pronóstico & Inf 80 & Sup 80 & Inf 95 & Sup 95 \\
\hline Ene & 2019 & 0.487060 & 0.304497 & 0.66962 & 0.207854 & 0.76627 \\
\hline Feb & 2019 & 0.420365 & 0.237213 & 0.60352 & 0.140258 & 0.70047 \\
\hline Mar & 2019 & 0.422562 & 0.238765 & 0.60636 & 0.141469 & 0.70365 \\
\hline Abr & 2019 & 0.544878 & 0.360377 & 0.72938 & 0.262708 & 0.82705 \\
\hline May & 2019 & 0.660712 & 0.475446 & 0.84598 & 0.377372 & 0.94405 \\
\hline Jun & 2019 & 0.780529 & 0.594436 & 0.96662 & 0.495924 & 1.06513 \\
\hline Jul & 2019 & 0.633562 & 0.446576 & 0.82055 & 0.347592 & 0.91953 \\
\hline Ago & 2019 & 0.600486 & 0.412540 & 0.78843 & 0.313048 & 0.88792 \\
\hline Sep & 2019 & 0.795259 & 0.606286 & 0.98423 & 0.506250 & 1.08427 \\
\hline Oct & 2019 & 0.642899 & 0.452829 & 0.83297 & 0.352212 & 0.93359 \\
\hline Nov & 2019 & 0.709260 & 0.518021 & 0.90050 & 0.416785 & 1.00174 \\
\hline Dic & 2019 & 0.807878 & 0.598937 & 1.01682 & 0.488330 & 1.12742 \\
\hline
\end{tabular}

Fuente: Elaboración propia

La representación gráfica del pronóstico obtenido permite visualizar el comportamiento probable en el tiempo del costo por peso de la Cadena Hotelera Cubanacán la cual se muestra en la figura 12.

\footnotetext{
“Visión de Futuro" Año 18, Volumen No 25 No 1, Enero - Junio 2021 - Pág 107-132

URL de la Revista: http://visiondefuturo.fce.unam.edu.ar/index.php/visiondefuturo/index

URL del Documento: https://visiondefuturo.fce.unam.edu.ar/index.php/visiondefuturo/issue/view/19

ISSN 1668 - 8708 - Versión en Línea

E-mail: revistacientifica@fce.unam.edu.ar
} 


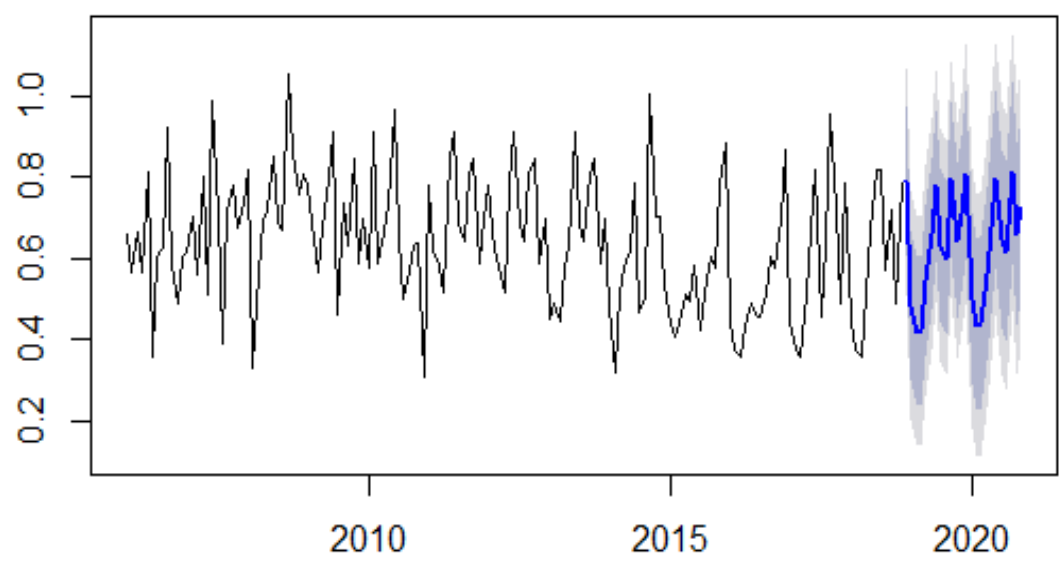

Figura N ${ }^{\circ}$ 12: Pronóstico del costo por peso mensual en la cadena hotelera Cubanacán para el año 2019 Fuente: R, versión 3.6.3

Luego de analizar las series demanda turística y costo por peso se hace necesario el análisis del indicador de eficacia: ingreso promedio mensual por turista, elemento este de gran importancia para cualquier organización. Por lo tanto, se estudia a continuación la serie temporal, a partir del año 2006 hasta el 2018 representada en la figura 13.

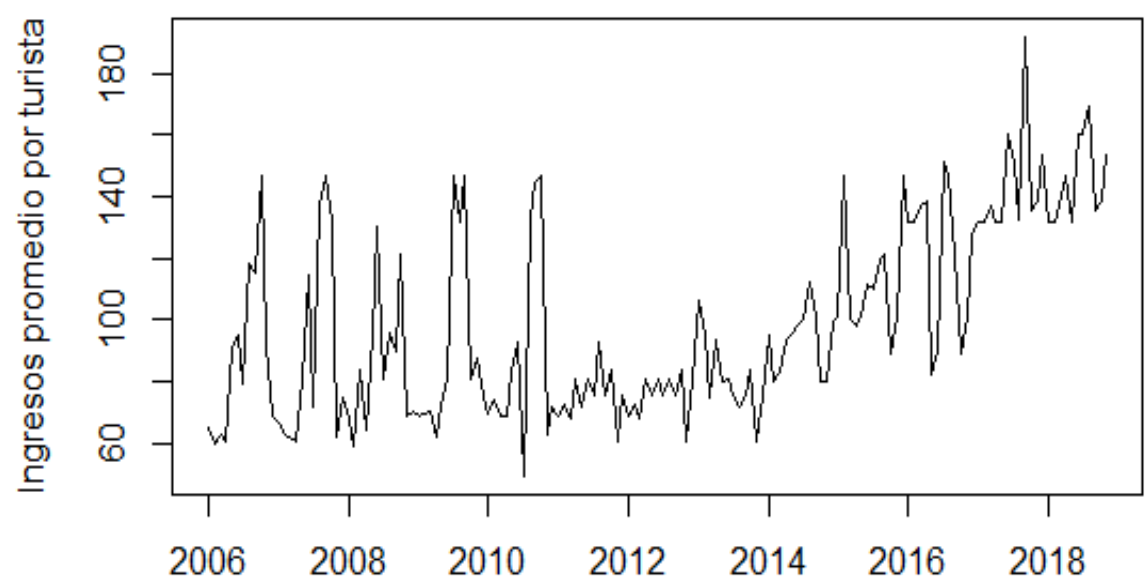

Figura $N^{\circ}$ 13: Serie temporal de los ingresos promedio por turista (Cadena Hotelera Cubanacán, 20062018)

Fuente: R, versión 3.6 .3

Se parte de la descomposición de la serie mediante el método de Loess, donde se observa poca influencia de la crisis económica ocurrida en el año 2008 y el paso de los huracanes Gustav e Ike, contrariamente a lo ocurrido con el resto de los indicadores estudiados con anterioridad. Existe un crecimiento exponencial de los ingresos promedio por turista a partir del año 2014, a pesar de la tendencia a disminuir el turismo, en esta cadena hotelera. Esto se debe a dos factores: la entrada de turistas de media y media alta clase, o sea, un turista más consumidor o a turistas que ingresan por primera vez al país. En el año 2017 se evidencia una pequeña fluctuación a causa del retroceso de las relaciones

\footnotetext{
"Visión de Futuro" Año 18, Volumen N²5 N 1, Enero - Junio 2021 - Pág 107-132

URL de la Revista: http://visiondefuturo.fce.unam.edu.ar/index.php/visiondefuturo/index

URL del Documento: https://visiondefuturo.fce.unam.edu.ar/index.php/visiondefuturo/issue/view/19

ISSN 1668 - 8708 - Versión en Línea

E-mail: revistacientifica@fce.unam.edu.ar
} 
diplomáticas Cuba - Estados Unidos referenciada en la figura 14.

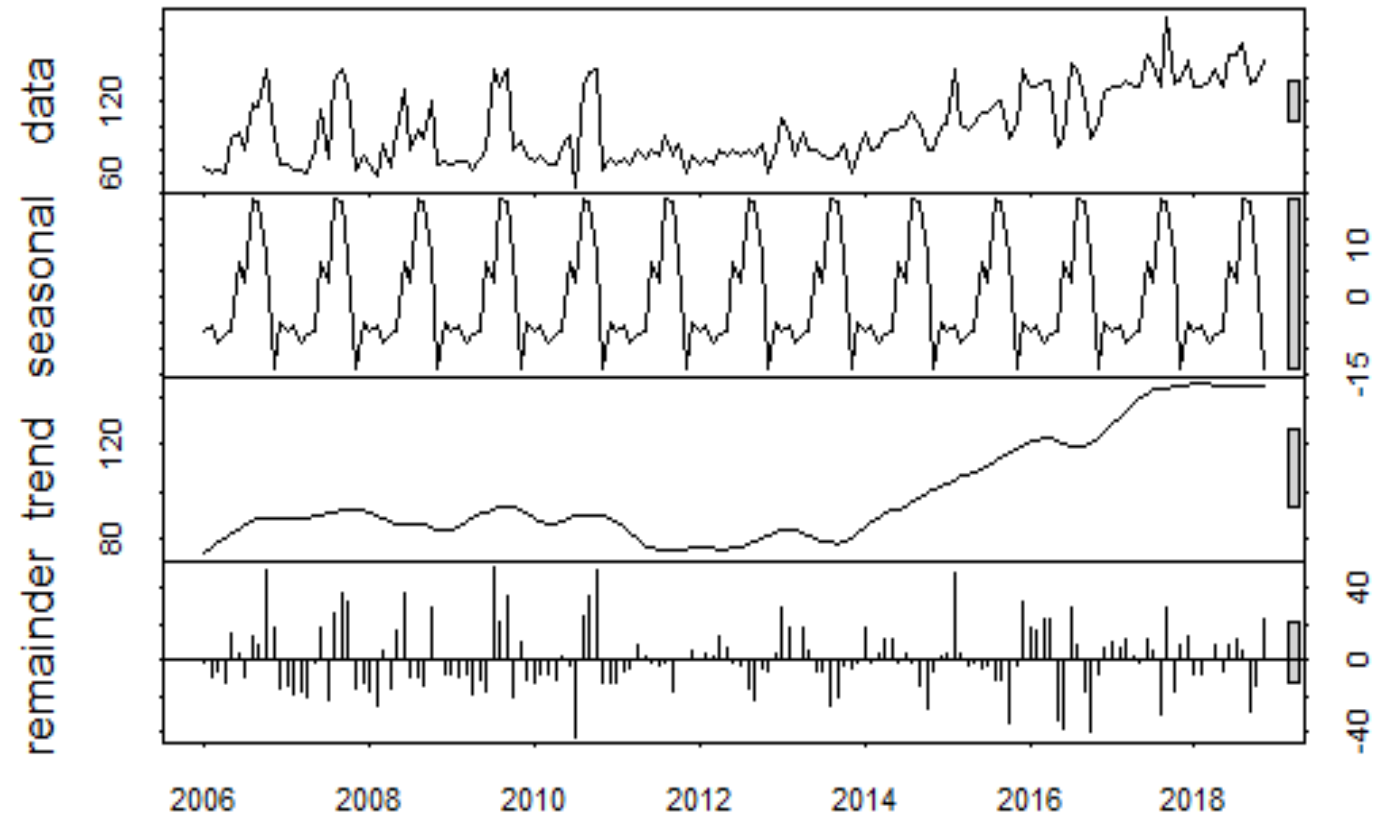

Figura № 14: Método de descomposición por Loess modelo aditivo para la serie temporal ingresos promedio por turista en la Cadena Hotelera Cubanacán

Fuente: R, versión 3.6.3

La observación del correlograma de la figura 15 muestra que la componente que predomina es la tendencia, pues los valores de autocorrelación van de valores positivos a negativos como se ilustra en la figura; no obstante, también está presente la componente estacional al evidenciarse que las autocorrelaciones presentan un máximo relativo con período 12

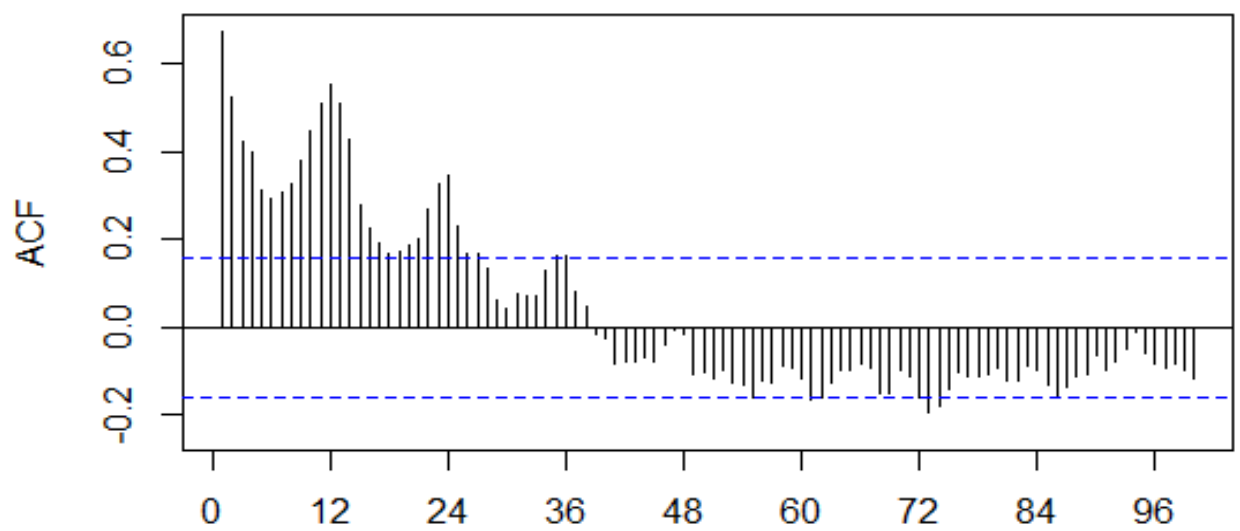

Figura N 15: Gráfica de la función de autocorrelación para la serie temporal ingresos promedio por turista en la Cadena Hotelera Cubanacán

Fuente: R, versión 3.6.3

Las características que son inherentes a la serie ingresos promedio por turista sugieren que la metodología más adecuada en este caso es el alisado exponencial, en particular el método Holt - Winters, que se emplea en series cronológicas que presentan tendencia y 
estacionalidad, elemento este ya explicado con anterioridad.

En la salida de $\mathrm{R}$ de la tabla 8 se tiene el modelo que mejor ajusta según la función automática del software, el cual selecciona dentro de los métodos de alisado exponencial el modelo matemático que contiene las menores medidas de dispersión.

Tabla No 8: Holt-Winters exponencial

\begin{tabular}{ccccccc}
\hline \multicolumn{7}{c}{ Coeficientes } \\
\hline Alpha & Beta & Gamma & $\mathrm{a}$ & $\mathrm{b}$ & $\mathrm{s} 1$ & $\mathrm{~s} 2$ \\
\hline 0.10341 & 0. & 0.4050 & 143.676 & 0.252 & 10.999 & 2.817 \\
\hline $\mathrm{s} 3$ & $\mathrm{~s} 4$ & $\mathrm{~s} 5$ & $\mathrm{~s} 6$ & $\mathrm{~s} 7$ & $\mathrm{~s} 8$ & $\mathrm{~s} 9$ \\
\hline 5.868 & 4.438 & 7.452 & -7.33 & 11.907 & 16.495 & 16.263 \\
\hline $\mathrm{s} 10$ & $\mathrm{~s} 11$ & $\mathrm{~s} 12$ & -2.066 & & \\
\hline 12.765 & -7.985 & MAE & MPE & MAPE & MASE \\
\hline ME & RMSE & Medidas de dispersión \\
\hline 1.37915 & 21.31734 & 15.60241 & -1.55655 & 16.01076 & 0.930843 \\
\hline \multicolumn{7}{c}{ Fuente: Elaboración propia }
\end{tabular}

Procediendo a demostrar la calidad del modelo, se realiza la prueba de contraste de Ljung-Box-Pierce que figura en la tabla 9, la cual permite corroborar el comportamiento de los residuos como ruido blanco ( $p$-valor $>0.05$ ).

Tabla No 9: Prueba de Ljung-Box

$\frac{\text { Box-Ljung test data: residuals }}{\mathrm{X} \text {-squared }=0.3719, \mathrm{df}=1, \mathrm{p} \text {-value }=0.542}$

Fuente: Elaboración propia

El análisis gráfico de los residuos permite ver que los mismos se comportan como ruido blanco. No hay relación de dependencia entre ellos como muestra la figura 16. 


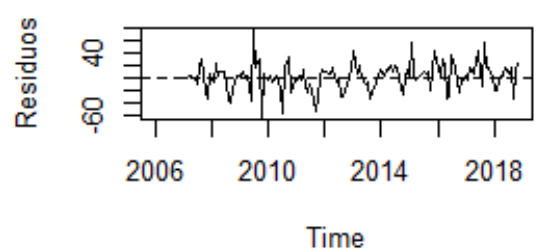

Series (Residuos)

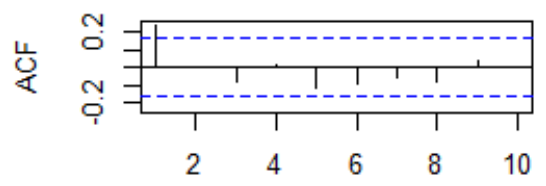

Lag

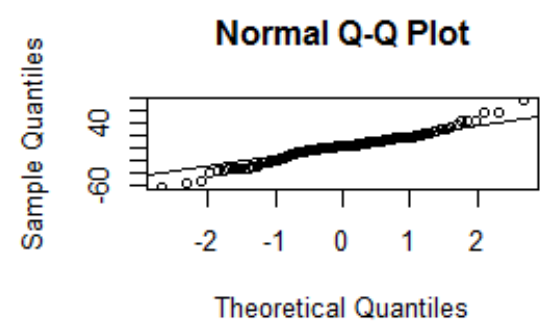

Histogram of Residuos

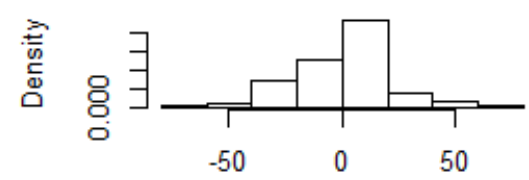

Residuos

Figura No 16: Gráfica de los residuos estandarizados del modelo de predicción Holt - Winters Fuente: R, versión 3.6.3

En la tabla 10, se presenta el pronóstico de los ingresos promedios por turista para el año 2019 en la Cadena Hotelera Cubanacán.

Tabla No 10: Pronóstico de la demanda turística para el año 2019

\begin{tabular}{ccccccc}
\hline & Año & Pronóstico & Inf 80 & Sup 80 & Inf 95 & Sup 95 \\
\hline Ene & 2019 & 146.996 & 119.493 & 174.500 & 104.933 & 189.060 \\
\hline Feb & 2019 & 150.299 & 122.650 & 177.947 & 108.013 & 192.584 \\
\hline Mar & 2019 & 149.121 & 121.328 & 176.914 & 106.615 & 191.627 \\
\hline Abr & 2019 & 152.387 & 124.450 & 180.323 & 109.661 & 195.112 \\
\hline May & 2019 & 137.857 & 109.777 & 165.937 & 94.913 & 180.801 \\
\hline Jun & 2019 & 157.345 & 129.123 & 185.567 & 114.183 & 200.507 \\
\hline Jul & 2019 & 162.185 & 133.822 & 190.549 & 118.807 & 205.563 \\
\hline Ago & 2019 & 162.205 & 133.701 & 190.709 & 118.612 & 205.798 \\
\hline Sep & 2019 & 158.958 & 130.314 & 187.602 & 115.151 & 202.766 \\
\hline Oct & 2019 & 138.460 & 109.676 & 167.243 & 94.439 & 182.481 \\
\hline Nov & 2019 & 144.631 & 115.708 & 173.553 & 100.398 & 188.863 \\
\hline Dic & 2019 & 157.947 & 126.334 & 189.561 & 109.598 & 206.297 \\
\hline
\end{tabular}

Fuente: Elaboración propia

Seguidamente, la figura 17 contiene la representación gráfica de las predicciones para el año 2019. En la misma se puede observar una variabilidad constante para el pronóstico en cuestión. 


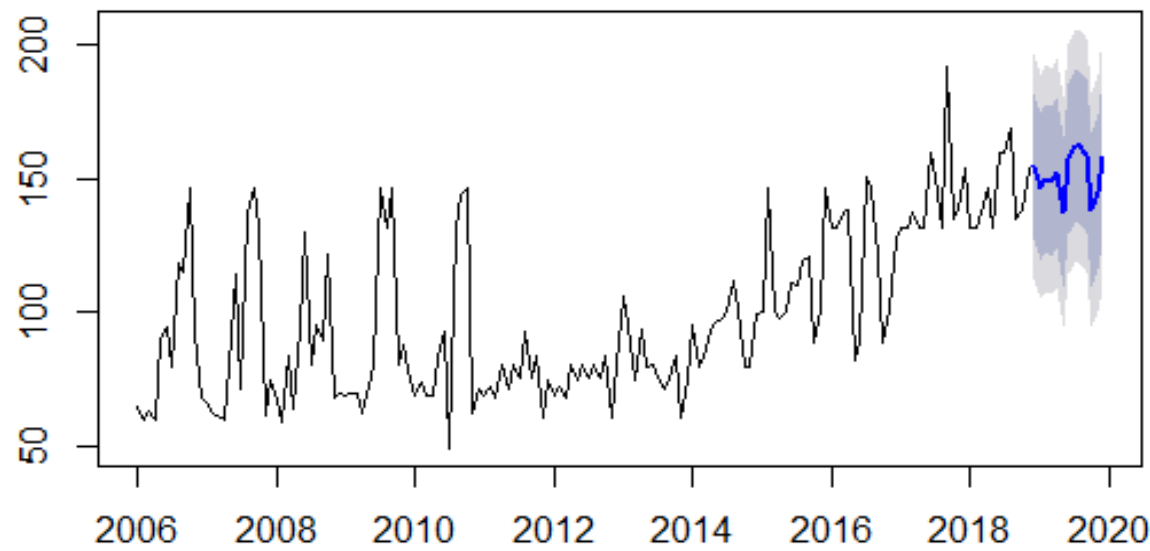

Figura № 17: Pronóstico del ingreso promedio por turista mensual en la Cadena Hotelera Cubanacán para el año 2019

Fuente: R, versión 3.6.3

Una vez presentados los modelos de pronóstico para las series de eficiencia y eficacia, se prosigue a su análisis multivariado de manera que sea posible interpretar como se ha comportado la Cadena Hotelera Cubanacán en el tiempo y a su vez caracterizarla.

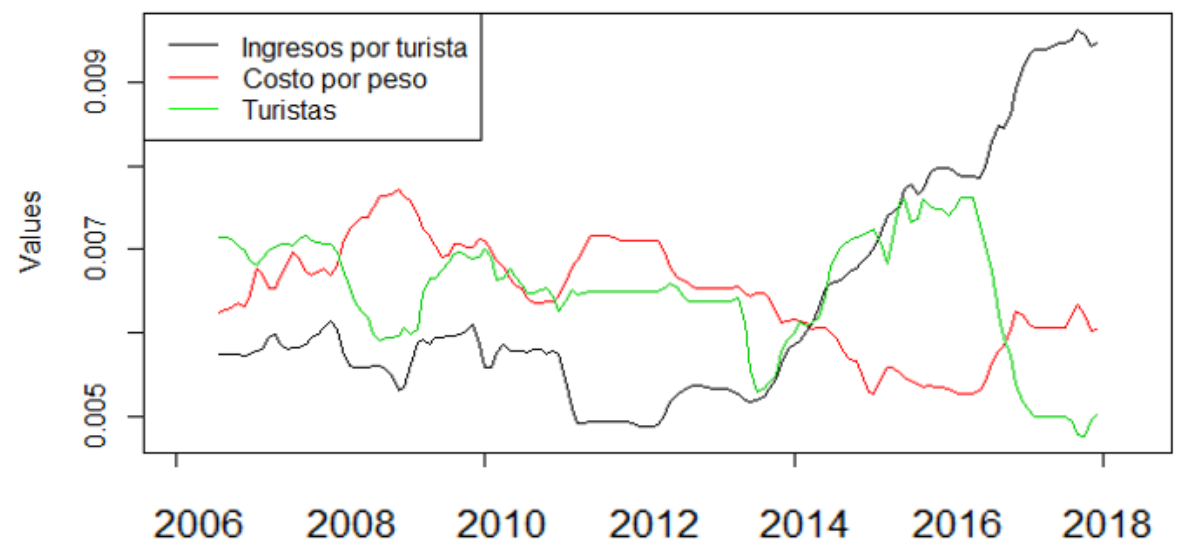

Figura No 18. Serie temporal multivariada de la demanda turística y los indicadores de eficiencia y eficacia (Cadena Hotelera Cubanacán, 2006-2018)

Fuente: R, versión 3.6.3

La representación multivariada contenida en la figura 18 constituye uno de los gráficos con mayor poder explicativo de la presente investigación. Esta permite apreciar la relación entre los tres elementos tomados como objeto de estudio: demanda turística, costo por peso e ingresos promedio por turista.

En la misma, se puede caracterizar el comportamiento de la Cadena Hotelera Cubanacán en cuatro etapas fundamentales: etapa de deficiencia en la gestión turística hasta el año 2008, en la cual ocurre una reducción de la actividad turística y por ende disminuyen los ingresos promedio por turista, sin embargo, se incrementa el costo por peso teniendo como significado un déficit en la gestión en la cadena hotelera. Etapa de recesión económica hasta el año 2014. Esta etapa se identifica por el efecto negativo causado por la crisis económica

\footnotetext{
"Visión de Futuro" Año 18, Volumen No 25 N 1, Enero - Junio 2021 - Pág 107-132

URL de la Revista: http://visiondefuturo.fce.unam.edu.ar/index.php/visiondefuturo/index

URL del Documento: https://visiondefuturo.fce.unam.edu.ar/index.php/visiondefuturo/issue/view/19

ISSN 1668 - 8708 - Versión en Línea

E-mail: revistacientifica@fce.unam.edu.ar
} 
mundial a partir del año 2008, caracterizada además por la caída de los ingresos promedio por turista y una actividad turística relativamente constante, por otro lado, ocurre un aumento de la eficiencia. Etapa marcada por el restablecimiento de las relaciones oficiales entre CubaEstados Unidos, en 2016, que trajo como efecto positivo un aumento de la demanda turística y un incremento sustancial de la eficiencia y eficacia en este sector. Etapa de la ruptura a partir del año 2017, en este momento la demanda turística decae de forma significativa, pero los ingresos promedio por turista mensuales se incrementan y el costo por peso se mantiene relativamente constante, proyectando una adecuada efectividad en cuanto a la gestión turística.

\section{CONCLUSIÓN}

El pronóstico de la demanda es pertinente para interconectar de forma adecuada los sectores productivos o de servicio que de una forma u otra intervienen en la actividad turística, por lo cual, los modelos matemáticos de series temporales tienen que estar presentes para una adecuada planificación en esta actividad económica estratégica y así mejore la calidad del proceso de toma de decisiones. En este punto, el estudio logra los modelos matemáticos para diagnosticar la demanda turística en la Cadena Hotelera Cubanacán en cuanto a su eficiencia y eficacia. Se pronostica una caída brusca en este sector para los próximos años agravada por la situación económica que presenta el país, recrudeciéndose en el periodo de ruptura de las relaciones diplomáticas entre Cuba-Estados Unidos a partir del año 2017. A esto habría que agregarle las consecuencias negativas que los efectos de la pandemia COVID - 19 traen consigo para un corto y mediano plazos. En este contexto complejo, surge la necesidad de combinar los modelos de pronósticos con el diseño de escenarios probables, en los que estos puedan materializarse, que propicie la combinación de métodos cuantitativos con métodos cualitativos. Por último, se caracteriza el comportamiento de la gestión turística en la cadena hotelera en el tiempo en cuatro etapas: etapa de deficiencia en la gestión turística, etapa de recesión económica, etapa de las relaciones diplomática y etapa de ruptura.

\section{REFERENCIAS}

Al-Musaylh, M. S., Deo, R. C., Adamowski, J. F., \& Li, Y. (2018). Short-term electricity demand forecasting with MARS, SVR and ARIMA models using aggregated demand data in Queensland, Australia. Advanced Engineering Informatics. https://doi.org/doi.org/10.1016/j.aei.2017.11.002

Bakar, N. A., \& Rosbi, S. (2017). Autoregressive Integrated Moving Average (ARIMA) Model for Forecasting Cryptocurrency Exchange Rate in High Volatility Environment: A New

\footnotetext{
"Visión de Futuro" Año 18, Volumen No 25 N 1, Enero - Junio 2021 - Pág 107-132

URL de la Revista: http://visiondefuturo.fce.unam.edu.ar/index.php/visiondefuturo/index

URL del Documento: https://visiondefuturo.fce.unam.edu.ar/index.php/visiondefuturo/issue/view/19

ISSN 1668 - 8708 - Versión en Línea

E-mail: revistacientifica@fce.unam.edu.ar
} 
Insight of Bitcoin Transaction. International Journal of Advanced Engineering Research and Science (IJAERS), 4(11). https://dx.doi.org/10.22161/ijaers.4.11.20

Barbosa, A. J., \& Gutiérrez, O. P. (2010). Propuesta de un procedimiento para el análisis de la demanda turística. TURyDES, 3(7).

Box, G. E., Jenkins, G. M., \& Reinsel, G. C. (1970). Time Series Analysis: Forecasting and Control. John Wiley \& Sons, Inc.

Brown, R. G. (1959). Statistical forecasting for inventory control. McGraw/Hil.

Castillo, L. A. F. (2017). Forecast of the National Index of Consumer Prices. Ricea: Revista Iberoamericana de Contaduría, Economía y Administración, 6(12). https://doi.org/10.23913/ricea.v6i12.95

Castro, A. D., \& Fernández, R. M. (2014). Pronóstico de la demanda turística hacia Cuba considerando el impacto del cambio climático. Revista Caribeña de Ciencias Sociales. https://econpapers.repec.org/article/ervrccsrc/y_3a2014_3ai_3a2014_5f08_3a01.htm

Crouch, G. I. (1994). The Study of International Tourism Demand: A Survey of Practice. Journal of Travel Research, 32(4), 41-55. https://doi.org/10.1177/004728759403200408

Cruz-Rodríguez, D. S., \& González-Laucirica, Á. M. (2014). Turismo senior: Análisis del comportamiento de las edades de los clientes que visitan el hotel $x$. varadero, cuba. RES NON VERBA, 5, 11.

Díaz-Pompa, F., Leyva-Fernández, L. de la C., Pérez, O. L. O., \& Mulet, Y. S. (2020). El turismo rural sostenible en Holguín. Estudio prospectivo panorama 2030. El Periplo Sustentable, 38, 174-193. https://doi.org/10.36677/elperiplo.v0i38.9265

Feng, Y., Li, G., Sun, X., \& Li, J. (2019). Forecasting the number of inbound tourists with Google Trends. Procedia Computer Science, 162, 628-633. https://doi.org/10.1016/j.procs.2019.12.032

Gómez, A. L. S. (2012). El pronóstico de la demanda turística incluyendo variables mercadológicas. Turismo y Desarrollo Local, 12.

Hącia, E. (2019). The role of tourism in the development of the city. Transportation Research Procedia, 39, 104-111. https://doi.org/10.1016/j.trpro.2019.06.012

Holt, C. C. (1957). Forecasting seasonals and trends by exponentially weighted averages. Office of Naval Research.

Jadhav, V., Reddy, B. V. C., \& Gaddi, G. M. (2017). Application of ARIMA Model for Forecasting Agricultural Prices. Journal of Agricultural Science and Technology, 19(5), 981-992.

Li, C., Ge, P., Liu, Z., \& Zheng, W. (2020). Forecasting tourist arrivals using denoising and potential factors. Annals of Tourism Research, 83, 102943. https://doi.org/10.1016/j.annals.2020.102943 
Li, G., Song, H., \& Witt, S. F. (2005). Recent developments in econometric modeling and forecasting. Journal of Travel Research, 44(1), 82-99.

Li, S., Chen, T., Wang, L., \& Ming, C. (2018). Effective tourist volume forecasting supported by PCA and improved BPNN using Baidu index. Tourism Management, 68, 116-126. https://doi.org/10.1016/j.tourman.2018.03.006

Liu, H., Li, C., Shao, Y., Zhang, X., Zhai, Z., Wang, X., Qi, X., Wang, J., Hao, Y., Wu, Q., \& Jiao, M. (2020). Forecast of the trend in incidence of acute hemorrhagic conjunctivitis in China from 2011-2019 using the Seasonal Autoregressive Integrated Moving Average (SARIMA) and Exponential Smoothing (ETS) models. Journal of Infection and Public Health, 13(2), 287-294. https://doi.org/10.1016/j.jiph.2019.12.008

Madrazo, L. M. R., Campdesuñer, R. P., Hernández, M. E. N., \& Ferrer, J. G. (2009). Modelo y procedimiento para la gestión de la demanda turística. Ciencias Holguín, 15(3), 1-12.

Milenković, M., Švadlenka, L., Melichar, V., Bojović, N., \& Avramović, Z. (2018). Sarima modelling approach for railway passenger flow forecasting. Transport, 33(5), 1113-1120. https://doi.org/10.3846/16484142.2016.1139623

Petrevska, B. (2017). Predicting tourism demand by A.R.I.M.A. models. Economic Research$\begin{array}{lll}\text { Ekonomska } \quad \text { Istraživanja, } & \text { 939-950. }\end{array}$ https://doi.org/10.1080/1331677X.2017.1314822

Rahman, A., \& Ahmar, A. S. (2017). Forecasting of primary energy consumption data in the United States: A comparison between ARIMA and Holter-Winters models. American Institute of Physics. http://dx.doi.org/10.1063/1.5002357

Song, H., \& Li, G. (2008). Tourism demand modelling and forecasting-A review of recent research. Tourism 203-220. https://doi.org/10.1016/j.tourman.2007.07.016

Song, H., Qiu, R. T. R., \& Park, J. (2019). A review of research on tourism demand forecasting: Launching the Annals of Tourism Research Curated Collection on tourism demand forecasting. Annals of Tourism Research, 75, 338-362. https://doi.org/10.1016/j.annals.2018.12.001

Su, Y., Gao, W., Guan, D., \& Su, W. (2018). Dynamic assessment and forecast of urban water ecological footprint based on exponential smoothing analysis. Journal of Cleaner Production, 195, 354-364. https://doi.org/10.1016/j.jclepro.2018.05.184

Winters, P. R. (1960). Forecasting Sales by Exponentially Weighted Moving Averages. Management Science, 6(3), 324-342. https://doi.org/10.1287/mnsc.6.3.324

Wu, D. C., Song, H., \& Shen, S. (2017). New developments in tourism and hotel demand modeling and forecasting. International Journal of Contemporary Hospitality 
Management, 29(1), 507-529. https://doi.org/10.1108/IJCHM-05-2015-0249

Xie, G., Qian, Y., \& Wang, S. (2020). A decomposition-ensemble approach for tourism $\begin{array}{lllll}\text { forecasting. Annals of } & \text { Tourism Research, }\end{array}$ https://doi.org/10.1016/j.annals.2020.102891

\section{RESUMEN BIOGRÁFICO}

\section{Reinier Fernández López}

Ingeniero Industrial por la Universidad de Pinar del Río "Hermanos Saíz Montes de Oca"/Upr, Cuba. Es Máster en Ingeniería Industrial y Sistemas por la Universidad Tecnológica de La Habana "José Antonio Echeverría" /Cujae, Cuba, en 2019. Profesor Asistente e investigador del Departamento de Matemática de la Facultad de Ciencias Técnicas en la Upr en la especialidad de Matemática Aplicadas. Es jefe del proyecto de innovación "Herramientas para la medición de la sostenibilidad y competitividad de los destinos turísticos de Pinar del Río". Línea de investigación: Matemática Aplicada a la Gestión Turística. No. ORCID: 0000-0003-1974-9209

\section{José Alberto Vilalta-Alonso}

Ingeniero Industrial. Máster en Aseguramiento de la Calidad. Es Dr. en Ciencias Técnicas por la Universidad Tecnológica de La Habana "José Antonio Echeverría" /Cujae, Cuba, en 2008. Profesor Titular del Departamento de Ingeniería Industrial de la Facultad de Ingeniería Industrial de la Cujae. Coordinador del programa de doctorado en Ingeniería Industrial y Sistemas. Es presidente de la Comisión Nacional de Carrera de Ingeniería Industrial de la República de Cuba. Línea de investigación: Estadística y Gestión de la Calidad. No. ORCID: 0000-0001-7505-8918

\section{Arely Quintero Silverio}

Licenciada en Economía. Es Dr. en Ciencias Técnicas por la Universidad de Pinar del Río "Hermanos Saíz Montes de Oca"/Upr, Cuba, en 2001. Profesor Titular del Departamento de Matemática de la Facultad de Ciencias Técnicas de la Upr. Metodóloga de Postgrado de la Upr. Miembro del Tribunal Permanente para el otorgamiento del Grado Científico de Doctor en Ciencias Técnicas (Geología). Línea de investigación: Matemática Aplicada a los Procesos Geológicos. No. ORCID: 0000-0003-29518957

\section{Ledy Raúl Díaz-González}

Ingeniero en Telecomunicaciones y Electrónica. Máster en Nuevas tecnologías de la informática y las Comunicaciones por la Universidad de Pinar del Río "Hermanos Saíz Montes de Oca"/Upr, Cuba, en 2007. Profesor Auxiliar del Departamento de Matemática de la Facultad de Ciencias Técnicas de la Upr. Jefe del Departamento de Matemática de la Facultad de Ciencias Técnicas de la Upr. Línea de investigación: Matemática Aplicada. No. ORCID: 0000-0002-8923-764X

\footnotetext{
"Visión de Futuro" Año 18, Volumen No 25 N 1, Enero - Junio 2021 - Pág 107-132

URL de la Revista: http://visiondefuturo.fce.unam.edu.ar/index.php/visiondefuturo/index

URL del Documento: https://visiondefuturo.fce.unam.edu.ar/index.php/visiondefuturo/issue/view/19

ISSN 1668 - 8708 - Versión en Línea

E-mail: revistacientifica@fce.unam.edu.ar
} 